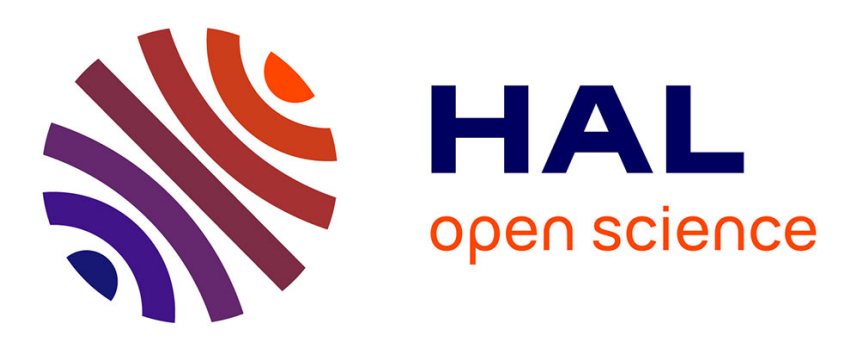

\title{
Dislocations and plasticity in semiconductors. I - Dislocation structures and dynamics
}

\author{
A. George, J. Rabier
}

\section{To cite this version:}

A. George, J. Rabier. Dislocations and plasticity in semiconductors. I - Dislocation structures and dynamics. Revue de Physique Appliquée, 1987, 22 (9), pp.941-966. 10.1051/rphysap:01987002209094100 . jpa-00245655

\section{HAL Id: jpa-00245655 https://hal.science/jpa-00245655}

Submitted on 1 Jan 1987

HAL is a multi-disciplinary open access archive for the deposit and dissemination of scientific research documents, whether they are published or not. The documents may come from teaching and research institutions in France or abroad, or from public or private research centers.
L'archive ouverte pluridisciplinaire HAL, est destinée au dépôt et à la diffusion de documents scientifiques de niveau recherche, publiés ou non, émanant des établissements d'enseignement et de recherche français ou étrangers, des laboratoires publics ou privés. 


\title{
REVUE DE PHYSIQUE APPLIQUÉE
}

Revue Phys. Appl. 22 (1987) 941-966

SEPTEMBRE 1987, PAGE 941

Classification

Physics Abstracts

$61.70-62.20 \mathrm{~F}$

\section{Dislocations and plasticity in semiconductors. I - Dislocation structures and dynamics}

\author{
A. George (*) and J. Rabier (**) \\ (*) Laboratoire de Physique du Solide, Unité Associée au CNRS n ${ }^{\circ} 155$, Ecole des Mines de Nancy, Institut \\ National Polytechnique de Lorraine, 54042 Nancy Cedex, France
}

(**) Laboratoire de Métallurgie Physique, Unité Associée au CNRS n 131, Faculté des Sciences, 40, avenue du Recteur Pineau, 86022 Poitiers Cedex, France

(Reçu le 18 décembre 1986, révisé le 4 mai 1987, accepté le 26 mai 1987)

\section{Overview article}

\begin{abstract}
Résumé. - Les structures de cœur des dislocations dans les semi-conducteurs élémentaires (ESC) et les composés semi-conducteurs (CSC) III-V sont discutées en relation avec les analogies qu'elles présentent avec celles des métaux cfc. On met l'accent sur les propriétés spécifiques des dislocations dans les SC aux températures plus basses que $0,6 T_{\mathrm{f}}$ où le glissement des dislocations est régi par un mécanisme de Peierls qui témoigne de la liaison covalente entre atomes. On fait une revue de la dynamique des dislocations en rapport avec des effets métallurgiques classiques ou des effets spécifiques aux SC associés aux niveaux électroniques introduits par les dislocations dans la bande interdite. Les analogies et les différences entre les structures et les propriétés des dislocations dans les ESC et CSC sont aussi discutées.
\end{abstract}

\begin{abstract}
Dislocation core structures in elemental semiconductors (ESC) and III-V compound semiconductors (CSC) with zinc blende structure are discussed in relation to their analogy with fcc metals. Emphasis is also put on the specific features of dislocations in SC at temperatures lower than $0,6 T_{\mathrm{m}}$ when dislocation glide is controlled by the Peierls mechanism reflecting the strong covalent bonding of the atoms. Dislocation dynamics relevant to classical metallurgical effects or to specific effects associated with electronic levels introduced by dislocations in the band gap are reviewed. Analogies and differences between dislocation structures and properties in ESC and CSC are also discussed.
\end{abstract}

\section{Introduction.}

The plasticity of semiconducting materials has been extensively studied for nearly thirty years : the purity and perfection of available crystals, the strong temperature dependence of the flow stress which allowed the behaviour to be changed from totally brittle to ductile and the possibility of applying many imaging techniques (etch pitting, X-ray topography, TEM) to the observation of dislocations have long promoted germanium (more easily deformed at moderate temperature), and later silicon, as model substances. These materials are well suited to the problem of connecting single dislocation velocity to the macroscopic mechanical behaviour in creep or constant strain rate tests. The very detailed review article of Alexander and Haasen appeared in
1968 [1], and although many questions were left open, it had a very impressive internal consistency, giving the idea that every thing of importance had been firmly established and well understood. However many papers have appeared more recently bringing up a lot of sometimes really surprising new findings, which have proved that these « model » substances are very complex.

The purpose of this review is to summarize experimental facts that are presently widely accepted, to describe recent results (with some emphasis on those obtained by the present authors and their coworkers !) and to underline some of the numerous questions which are still matter of controversy. Its scope is restricted to mechanical properties, i.e. dislocations, their mobilities and the way they interact with other defects. Electrical and optical proper- 
ties of dislocations will be dealt with only when they affect the mechanical behaviour.

We have chosen to focus our attention on elemental semiconductors (ESC) and III-V compounds (CSC), although II-VI compounds seem to have very promising applications and show interesting effects such as the photoplastic effect. Some special attention is paid to the relation between microscopical features of dislocation structures and their dynamic and macroscopic behaviour.

In many ways covalent crystals are not very special but behave just like metals. The well known mechanical behaviour of fcc or bcc metals offers a convenient reference or a guideline for a qualitative comparison and it will be emphasized how much and in what sense the plastic behaviour of covalent semiconductors is like that of metals, although in many instances a similar phenomenological behaviour results from rather different microscopic origins.

For brevity the reader will sometimes be referred to recent review papers, rather than the original papers.

This paper is organized in two main parts. In the first part the structural characteristics and dynamic properties of single dislocations are presented. The second part is concerned with the way their properties are reflected in the plastic deformation of semiconductor crystals.

In this first art considerati res of dislocations are given first as a way to introduce the notion of antiphase reconstruction defects or " solitons », then a brief outline of models of dislocation glide is presented. Dislocation-point defects interactions are then treated and dislocation velocity measurements are reported at the end of the paper, with emphasis on experimental findings that are not satisfactorily explained by simple models.

\section{Dislocation structure.}

2.1 CRySTAL STRUCTURE AND DISLOCATIONS. Tetravalent SC crystallize in the diamond structure : a fcc lattice whose basis is built with two atoms located at $(0,0,0)$ and $(1 / 4,1 / 4,1 / 4)$. Each of the atoms is bonded to its four nearest neighbours located at the summits of a tetrahedron by a $(\mathrm{sp})^{3}$ covalent bond (Fig. 1a).

Most of the III-V and II-VI A-B CSC crystallize in the zinc blende structure which is analogous to the diamond structure but the basis of the fcc lattice is now built with two different atoms : $A$ at $(0,0,0) \mathrm{B}$ at $(1 / 4,1 / 4,1 / 4)$. Each of the atoms possesses four nearest neighbours of the other type at the summits of the tetrahedron (Fig. 2a). Since the two atom types show a different electronic affinity the covalent bond has now a partial ionic character.

Table I lists the principal characteristics of ESC and CSC useful in this paper. CSC have been

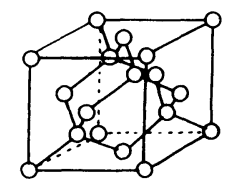

a

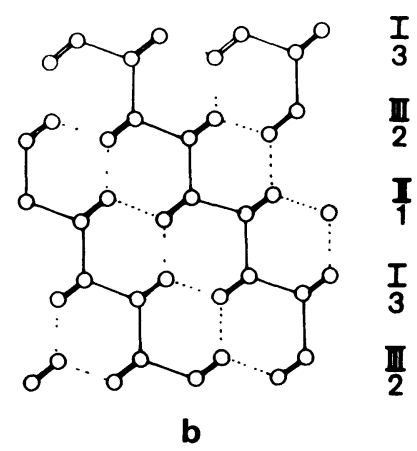

Fig. 1. - The diamond structure of tetravalent elemental semiconductors (ESC). (a). - unit cell. (b). - projection on (110) : two (110) planes are represented, full lines link atoms located in the (110) plane at $z=0$, broken lines link atoms located in the (110) plane at $z=-a_{0} \sqrt{2} / 4$, double lines link atoms of these two (110) planes.
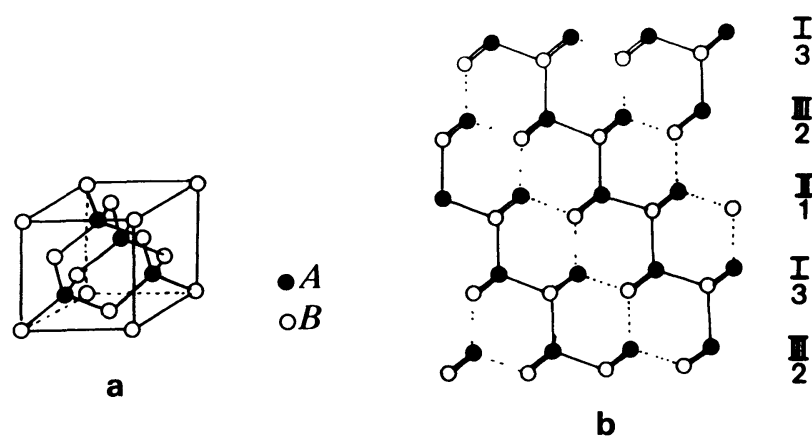

Fig. 2. - The zinc blende structure of A B compound semiconductors (CSC), (a). - unit cell. (b). - projection on (110) with the different nature of the atoms $A$ and $B$ (open and filled circles). The atomic positions in the two represented (110) planes are the same as in Fig. 1 (b).

classified as a function of their ionicity factor $f_{\mathrm{i}}$ defined by Phillips [2]. Room temperature elastic properties used in dislocation theory are given and compared to those of typical fcc and bcc metals. Isotropic elastic theory strictly applies when $A$ (anisotropy factor for elasticity) equals one.

As for fcc metals, the glide system for ESC and CSC is $a / 2$ [110] (111). It is then convenient to describe dislocations in SC starting from a projection of the diamond structure (or the zinc blende structure) on the (110) plane. The stacking order parallel to (111) is deduced from that of a fcc lattice I/II/III * by doubling the atoms in the stacking sequence : I1/II2/III3. In CSC the nature of the atoms is different in each of the consecutive planes of the (111) stacking (i.e. I(A), 1(B) in Fig. 2b). In most

(*) This convention is used instead of the standard $A / B / C$ convention for fcc metals in order to avoid confusions with the $\mathrm{A}, \mathrm{B}$ natures of the atoms and dislocations in CSC. 
Table I. - Some physical characteristics of semiconductor and elastic properties compared to metals at room temperature.

\begin{tabular}{|l|c|c|c|c|c|c|c|c|c|c|}
\hline & $f_{\mathrm{i}}$ & $\begin{array}{c}E_{\mathrm{g}} \\
\mathrm{eV}\end{array}$ & $\begin{array}{c}a_{\mathrm{o}} \\
\AA\end{array}$ & $\begin{array}{c}b \\
\AA\end{array}$ & $\begin{array}{c}T_{\mathrm{m}} \\
{ }^{\circ} \mathrm{C}\end{array}$ & $\begin{array}{c}\mu_{10} \\
10^{\mathrm{Pa}}\end{array}$ & $\begin{array}{c}A \\
\mathrm{eV}\end{array}$ & $\begin{array}{c}\mu b^{2} \\
\mathrm{eV} / \AA\end{array}$ & $\begin{array}{c}\mu b \\
\mathrm{~J} / \mathrm{m}^{2}\end{array}$ \\
\hline $\mathrm{Si}$ & 0 & 1.14 & 5.431 & 3.84 & 1420 & 6.8 & 1.64 & 24.07 & 6.26 & 26.11 \\
\hline $\mathrm{Ge}$ & 0 & 0.67 & 5.657 & 4.00 & 937 & 5.6 & 1.66 & 22.4 & 5.60 & 22.4 \\
\hline $\mathrm{CaSb}$ & 0.261 & 0.72 & 6.095 & 4.31 & 706 & 3.5 & 1.76 & 17.51 & 4.06 & 15.08 \\
\hline $\mathrm{GaAs}$ & 0.310 & 1.42 & 5.653 & 4.00 & 1238 & 4.8 & 1.82 & 19.16 & 4.79 & 19.18 \\
\hline $\mathrm{InSb}$ & 0.321 & 0.17 & 6.479 & 4.50 & 530 & 2.5 & 1.82 & 15.02 & 3.27 & 11.45 \\
\hline $\mathrm{GaP}$ & 0.327 & 2.26 & 5.449 & 3.85 & 1467 & 5.8 & 1.79 & 20.73 & 5.38 & 22.34 \\
\hline InAs & 0.357 & 0.36 & 6.058 & 4.28 & 942 & 3.1 & 2.08 & 15.23 & 3.55 & 13.28 \\
\hline InP & 0.421 & 1.35 & 5.868 & 4.15 & 1058 & 3.6 & 2.06 & 16.07 & 3.87 & 14.93 \\
\hline $\begin{array}{l}\text { Al } \\
\text { F.C.C. }\end{array}$ & - & - & 4.049 & 2.68 & 660 & 2.7 & 1.23 & 4 & 1.4 & 7.7 \\
\hline $\begin{array}{l}\text { Fe } \\
\text { B.C.C. }\end{array}$ & - & - & 2.863 & 2.48 & 1529 & 7.9 & 2.36 & 7.9 & 3.18 & 19.6 \\
\hline
\end{tabular}

$f_{\mathrm{i}}:$ ionicity factor

$E_{\mathrm{g}}$ : energy gap.

$b$ : Burgers i.ector.

$a_{0}:$ lattice parameter.

$T_{\mathrm{m}}:$ melting temperature.

$\mu$ : shear modulus.

$A: 2 \mathrm{C}_{44} / \mathrm{C}_{11}-\mathrm{C}_{12}$ : anisotropy factor for elasticity.

crystal structures, as well as fcc metals, the easy glide system satisfies the condition $b / d_{h k l}$ minimum [3] since the distortion induced by a dislocation of Burgers vector $b$, is spread out on a small distance $(b)$ between two widely spaced dense glide planes $\left(d_{h k l}\right)$. However this simple criterion does not apply for SC.

The glide shear may occur between the widely spaced planes $(I, 1)$ or between narrowly spaced ones (II, 1) (Fig. 1b, 2b). Then two dislocation core structures can be expected corresponding, respectively, to these two sets of dislocation movement : shuffle set (s) and glide set (g). It has long been believed that glide takes place in the shuffle mode since the number of covalent bonds to be broken is three times smaller than in the glide mode. However it has been demonstrated that dislocations are dissociated in ESC [4, 5] and CSC [6] and remain dissociated when moving $[7,8]$.
A perfect dislocation in the glide set can dissociate into two Shockley partials bounding a stacking fault in full analogy with fec metals following the reaction :

$a / 2[1 \overline{1} 0] \rightarrow a / 6[1 \overline{2} 1]+a / 6[2 \overline{1} \overline{1}]$ in the (111) plane.

This stacking fault can only be created in the glide set between two nearest planes. A dissociated dislocation in the shuffle set is more complex since a shuffle dislocation results from a shear between two planes 1 , I whereas the stacking fault lies necessarily between II, 1. This involves complex atomic movements in the core of a moving shuffle partial so that dissociated dislocation glide in the glide set is usually assumed.

However some results indicate a remarkable density of point defects $[9,10]$ produced by plastic deformation, suggesting that point defects may be 
lost by dislocations during their motion. This can be explained if the dislocation can absorb a line of point defects along the core of one of its partials following reaction : (cf. Fig. 3).

$$
\begin{aligned}
& \left\{\begin{array}{l}
a / 2[01 \overline{1}]+a / 6[1 \overline{2} 1]+a / 6[2 \overline{1} \overline{1}] \\
a / 2[0 \overline{1} 1]
\end{array}\right\} \rightarrow \\
& \left\{\begin{array}{l}
a / 6[11 \overline{2}]+a / 6[2 \overline{1} \overline{1}] \\
a / 2[0 \overline{1} 1]
\end{array}\right\}
\end{aligned}
$$

where the row of point defects is formally equivalent to the dipole $a / 2[01 \overline{1}]+a / 2[0 \overline{1} 1]$, with a one atom height.
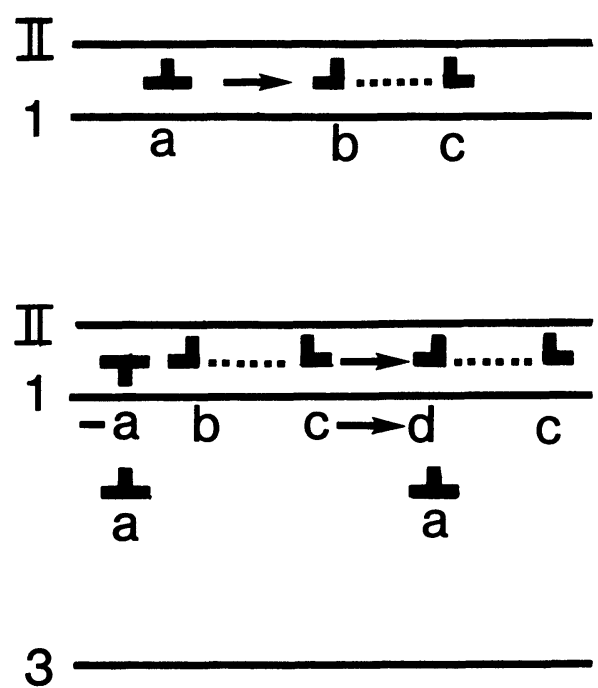

Fig. 3. - (a). - Dissociation in the glide set. $(a) \rightarrow(b)+(c)$. (b). - Interaction of a dissociated dislocation with a vacancy row.

The strain field of the resultant associated partial dislocation $(a / 6[11 \overline{2}]+a / 2[0 \overline{1} 1])$ is nearly the same as the $a / 6[1 \overline{2} 1]$ partial of reaction (1). Association of point defects can result from absorption of vacancies or interstitials on part of the length of the partial dislocation i.e. an elementary step of the climb mechanism. The glide of such a partial requires in addition to the displacement in the glide plane itself, a positive (or negative) elementary climb step followed by a negative (or positive) elementary climb step. This only implies the local migration of point defects during the dislocation movement without any need for the creation of point defects. However recent experiments [11] have shown the occurrence of point defects, left behind by a gliding dislocation, the concentration of which was found to be better correlated to shear strain than to dislocation density. This implies a climb component in the dislocation movement since emitted defects are not re-absorbed by dislocations to provide the opposite climb motion. A pure climb component can be understood in these conditions, if point defects clusters are formed when point defects are left behind by the dislocation so that they are not available for the opposite climb motion. However this concentration of point defects could also be explained by classical dislocation mechanisms : formation and breaking of dislocation dipoles...

Coming back to the glide and shuffle set problem there seems to exist a contradiction between the stationary dislocation in which the energy criterion yields a dissociation in the glide set and the moving dislocation for which the less bonds are broken when moving, the easiest the dislocation movement is, i.e. in the shuffle set. However this apparent contradiction seems to be avoided when reconstruction defects are introduced (antiphase defects or solitons) (see Sect. 2). In this theory kink nucleation and migration are achieved at antiphase defects or solitons without breaking additional bonds. Breaking bonds is not a problem any more so that dislocations can move and dissociate in the glide mode.

In what follows dislocation core configurations are referred to the glide set. However the coexistence of these two types of core structures cannot be excluded ; this may be of importance for the nucleation of double kinks [12].

A complication occurs in the description of dislocation cores from the existence of two types of atoms in CSC. In the two dislocation sets two $t$ es of can e defined following the nature A (B) of the atoms ending the extra-half plane of dislocations (Fig. 4). It is worth recalling

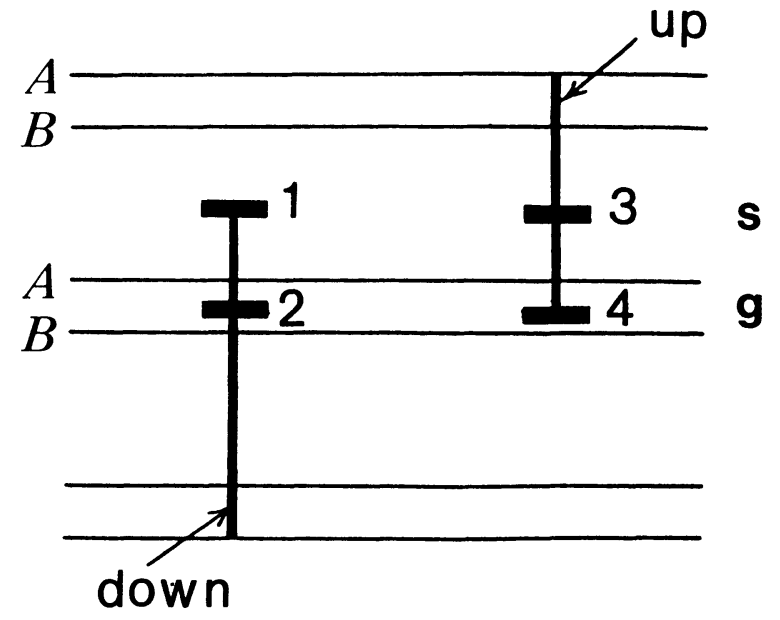

Fig. 4. - Different core structures of dislocations in CSC. The nature of the dislocation with the extra half-plane down can be: 1. Dislocation $A$ in the shuffle set: $A(s)$. 2. Dislocation $B$ in the glide set : B (g). The nature of the dislocation with the extra half-plane up can be : 3 . Dislocation B in the shuffle set : B(s). 4. Dislocation A in the glide set : $A(g)$. The assumption on the location of the dislocation core (glide or shuffle set) changes the nature of the atomic row ending the extra half-plane ( $\alpha$ dislocations $\mathrm{B}(\mathrm{g}), \boldsymbol{\beta}$ dislocations $\mathrm{A}(\mathrm{g}))$. 
that the nature $\mathrm{A}$ or $\mathrm{B}$ of dislocations is deduced

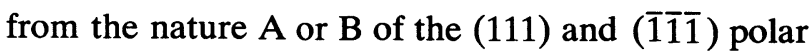
surfaces which induces different etching properties $\left({ }^{*}\right)$. Then the determination of position of the extra half-plane (up or down) is required and a particular dislocation set ( $\mathrm{g}$ or $\mathrm{s}$ ) has to be assumed. Several determinations and assumptions are required and confusions can occur. Many results have been interpreted on the assumption that slip takes place between the widely spaced (111) planes, i.e. shuffle set, so that dislocations with their extra half-plane ending on A or B atoms are called $\alpha$ or $(\mathrm{A}(\mathrm{s}))$ or $\beta$ (or B (s)) respectively. However since slip seems to occur in the glide set, dislocations previously assumed to be $\alpha$ and $\beta$ are actually $B(g)$ and $\mathrm{A}(\mathrm{g})$ respectively. These notations were recommanded at the Hünfeld Conference on dislocations in SC [14] (The $\alpha$ and $\beta$ terminology when used in what follows has the above meaning).

At low temperatures dislocations lie along the $\langle 110\rangle$ directions of the (111) glide plane. They correspond to the valleys of the Peierls potential. Figure 5a shows a perfect dislocation loop with the geometry of the extra half-planes. This shows that a glide loop possesses two types of dislocation segments, screw and $60^{\circ}$ dislocations $\left(60^{\circ}\right.$ is the angle between dislocation line and Burgers vector). The $60^{\circ}$ segments are of two different types $A$ and $B$ in CSC since extra half-planes of these dislocations are respectively up and down. The same representation for a dissociated dislocation shows two types of dislocation segments : $30^{\circ}$ and $90^{\circ}$ which can be associated with two types of dislocations $A$ and $B$ in CSC. In this latter case the stacking fault is sandwiched between $30^{\circ}-90^{\circ}, 90^{\circ}-30^{\circ}$ or $30^{\circ}-30^{\circ}$ partial dislocations (Fig. 5b).

The cores of these partials $\left(30^{\circ}\right.$ and $\left.90^{\circ}\right)$ are shown in figure 6. A row of dangling bonds is associated with the core of the $30^{\circ}$ partial and two rows with the $90^{\circ}$ partial. The cores of these partials are now believed to be reconstructed [15-17] : along the core for $30^{\circ}$ partials, with a doubling of the period, and across the core for $90^{\circ}$ partials (Fig. $6 \mathrm{~b}, \mathrm{c}$ ). Similar reconstruction of dislocation core in CSC is doubtful since the bounding on a A partial $\left(30^{\circ}\right.$ or $\left.90^{\circ}\right)$ would take place between two $\mathrm{A}$ atoms. This reconstruction should become more difficult as the ionic character of the compound increases. In GaAs, calculations by Jones et al. [18] suggest instead another kind of reconstruction with atom displacements in the core in order to shorten the distance between A and B atoms.

2.2 STACKING FAULT ENERGIES. - Dislocation splitting in SC has been investigated using the weak

(*) Convergent Beam Diffraction technique can also be used to determine the nature of the (111) surfaces [13].
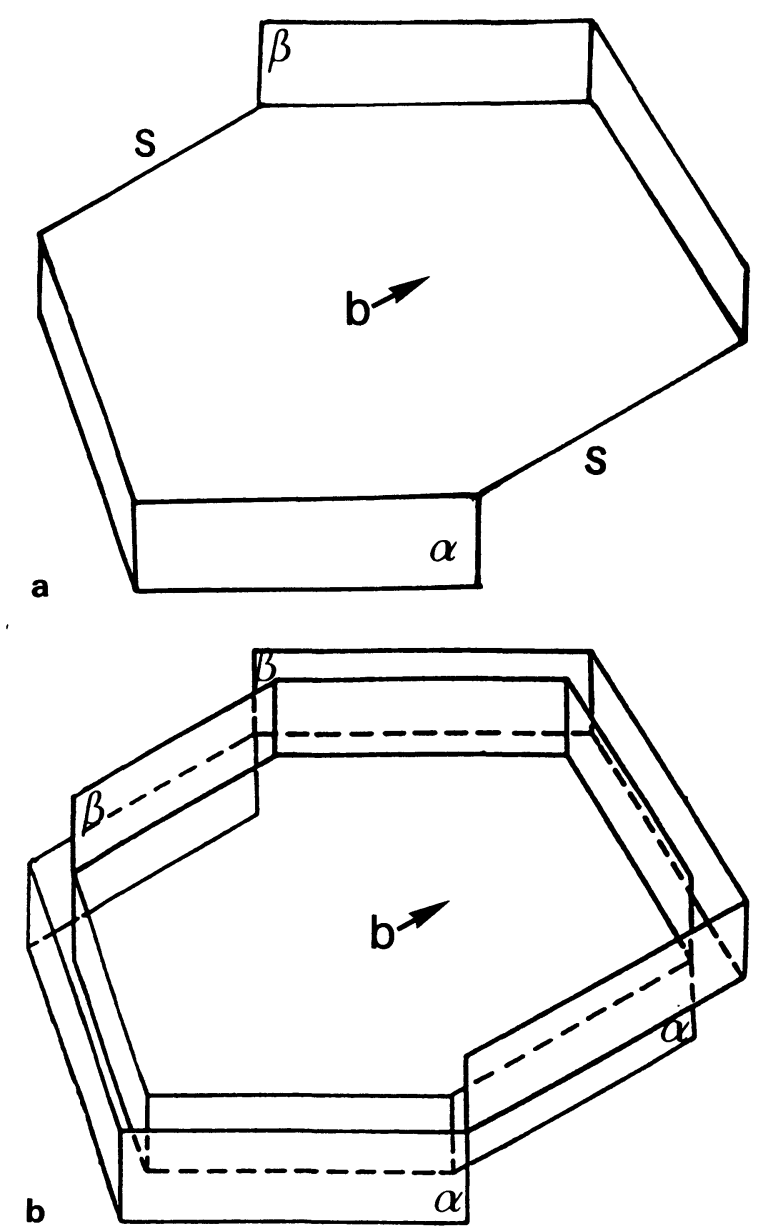

Fig. 5. - (a). - Perfect dislocation glide loop in the glide plane. Dislocation segments lie along $\langle 110\rangle$ directions in the glide plane. Extra half-planes are shown to give the $\alpha$ or $\beta$ character of the dislocation segments in CSC. (b). Dissociated dislocation glide loop in the (111) glide plane. Extra half-planes of each of the partials are shown in order to give the $\alpha$ or $\beta$ character of the partial dislocation segments in CSC.

beam technique and high resolution electron microscopy (HREM). Stacking fault values were deduced from measurements of splitting distances on isolated dislocations (ID), extended node configurations (EN), double ribbons of stacking faults (DR) as well as faulted dipoles (FD). Results of such investigations are reported in table II.

Dislocation splitting has only been widely investigated in $\mathrm{Si}, \mathrm{Ge}$ and $\mathrm{GaAs}$. Single dislocations created by plastic deformation in Si consist of two partials bounding an intrinsic stacking fault although extrinsic stacking fault energy $\gamma_{\mathrm{e}}$ is nearly equal or lower than intrinsic one $\gamma_{i}$ (cf. Tab. II). Intrinsic faults are also found in ESC and CSC. In GaAs Feuillet [30] found a slight difference between the stacking fault apparent energy in between A partials on one hand and B partials on the other hand. This cannot be explained by energy differences between the two types of stacking faults. 

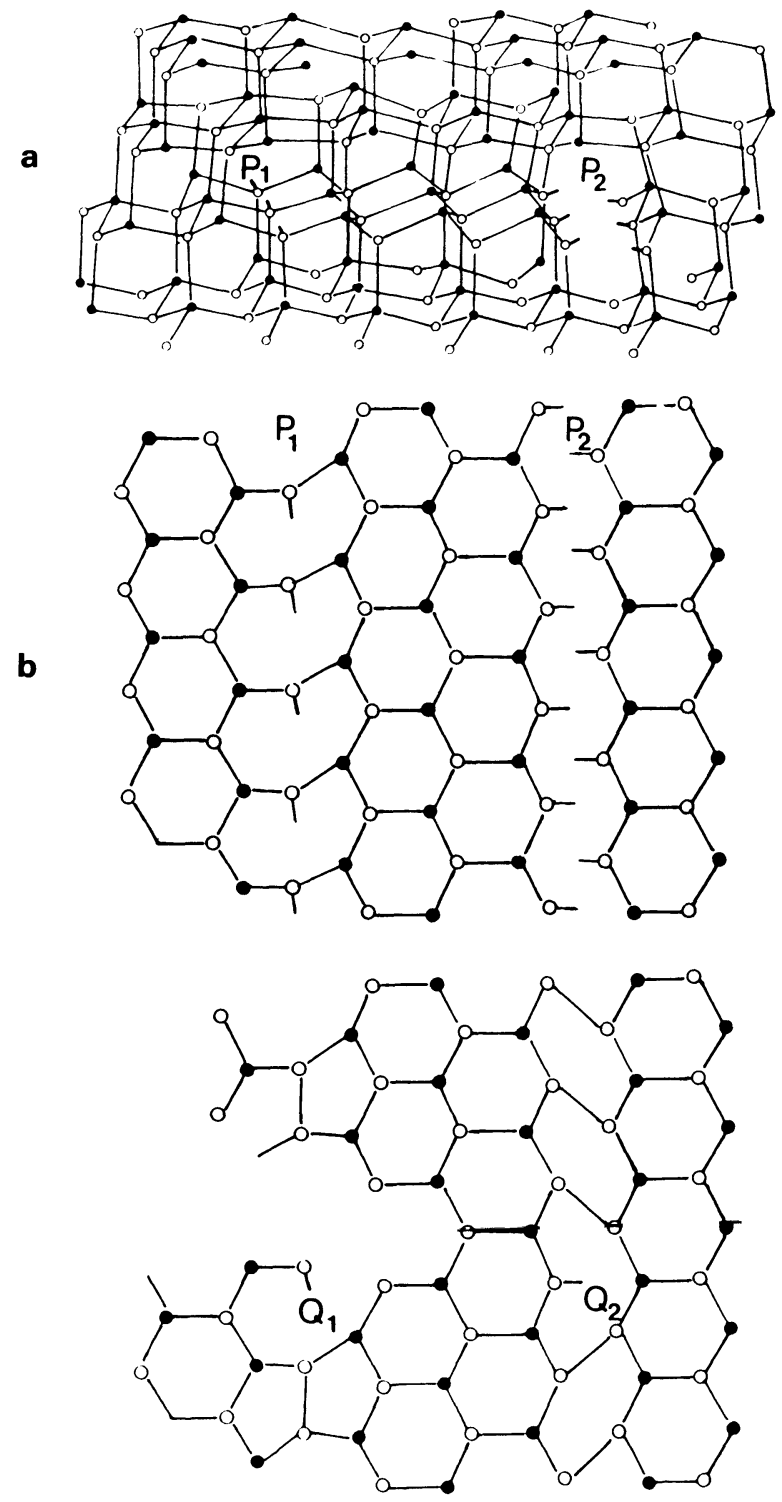

Fig. 6. - (a). $-60^{\circ}$ dislocation dissociated into $30^{\circ}(\mathrm{P} 1)$ and $90^{\circ}$ (P2) partial dislocations. Projection perpendicular to the dislocation line. (b). - Same configuration as in Fig. 6 (a) projected on the (111) glide plane. Atoms of planes 1 and II are shown. They have the same nature in ESC and different nature in CSC. Partial dislocations are not reconstructed. (c). - Same configuration as in Fig. 6 (b) but partial dislocations are now reconstructed. The reconstruction can be imperfect so that reconstruction defects exist. A reconstruction defect (soliton) in shown on each of the partials $\left(Q_{1}\right.$ on $P_{1}, Q_{2}$ on $\left.P_{2}\right)$.

In order to compare stacking fault energies in different CSC a reduced stacking fault energy $\gamma^{\prime}$ has been introduced by Gottschalk et al. [6]. $\gamma^{\prime}$ is related to a surface available for each A or B atom and can be written as $\gamma^{\prime}=\sqrt{3} / 4 a_{0}^{2} \gamma$ measured in meV/atom. This $\gamma^{\prime}$ energy decreases for CSC when the ionic character $f_{\mathrm{i}}$ of the bond increases. However this apparent consistency fails when data are compared to those of ESC where $f_{\mathrm{i}}=0$, (cf.
Tab. II) and those of II-VI compounds. No apparent consistency is also found when $\gamma$ is expressed in $\mu b$ units.

These observations show a good analogy between $\mathrm{SC}$ and fcc metals. Trends due to dislocation splitting are the same : extended node formation $[5,19,28]$, faulted dipoles [21, 24, 28], dislocation climb under irradiation [31, 32]. The covalent character of bonds in SC is not determining in the dislocation configurations when the temperature is high enough. In this range of temperature dislocation splitting should have the same consequences on SC plasticity as it has on fcc metals plasticity. However activation energies deduced from elastic interactions between dislocations must be scaled by the $\mu b^{3}$ factor which is greater in SC than in fcc metals (cf. Tab. I). Such an example can be given for the cross slip mechanism which requires a constriction of the stacking fault in the Friedel-Escaig model [32]. The activation energy calculated from this model is $7.4 \mathrm{eV}\left(T=1250^{\circ} \mathrm{C}\right)$ and $4.3 \mathrm{eV}\left(T=840^{\circ} \mathrm{C}\right)$ for Si and Ge respectively, whereas for various fcc metals the values range from $0.2 \mathrm{eV}$ to $1.4 \mathrm{eV}$ at room temperature [34]. The similar scaling with the $\mu b^{3}$ factor applies for climb of largely dissociated dislocations [31, 32] and any mechanism relevant to fcc metals.

\section{Mechanism of dislocation glide.}

3.1 ESC. - Dislocation glide at low temperature is controlled by the Peierls-Nabarro mechanism. A dislocation of Burgers vector, $b$ is transferred from one Peierls valley to the next one, distant by $h$, by the nucleation and migration of double kinks along the dislocation. Two cases have been discussed by Hirth and Lothe [35] :

- for small kink density or short dislocation segment of length $L$, the kinks can move along $L$ without being annihilated ; the dislocation velocity, $v$, is proportional to $L$ :

$$
v=h L J
$$

where $J$ is the nucleation rate of double kinks per unit length of dislocation

$$
J=\nu_{0} \frac{\tau b h}{k T} \exp \left(-2 F_{\mathrm{k}}+W_{\mathrm{M}}\right) / k T
$$

where $\nu_{0}$ is the attempt frequency of a kink, $\tau:$ the applied stress, $T$ : the temperature, $2 F_{\mathrm{K}}$ : the free enthalpy of formation of a double kink of critical length under $\tau, W_{\mathrm{M}}$ : the activation energy for kink migration

$$
v=L \tau b h^{2} / k T \nu_{0} \exp \left(-2 F_{\mathrm{k}}+W_{\mathrm{M}}\right) / k T
$$

- for high kink density or long dislocation segments, kinks are annihilated with kinks of opposite sign before reaching the ends of the dislocation 
Table II. - Stacking fault energies in S.C.

\begin{tabular}{|c|c|c|c|c|c|c|c|}
\hline \multicolumn{2}{|c|}{$\gamma_{\mathrm{i}} \mathrm{mJ} / \mathrm{m}^{2}$} & $\gamma_{\mathrm{e}} \mathrm{mJ} / \mathrm{m}^{2}$ & $\begin{array}{c}\text { Experimental } \\
\text { technique }\end{array}$ & $\begin{array}{c}\gamma_{\mathrm{i}}^{\prime} \\
\mathrm{meV} / \mathrm{at}\end{array}$ & $\begin{array}{l}\gamma_{\mathrm{i}} / \mu b \\
\times 10^{3}\end{array}$ & $\begin{array}{l}\gamma_{\mathrm{i}}^{\prime} / \mu b \\
\times 10^{3}\end{array}$ & $f_{\mathrm{i}}$ \\
\hline $\mathrm{Si}$ & $\begin{array}{r}51 \pm 5 \\
50 \pm 15 \\
71 \pm 16 \\
58 \pm 13 \\
70 \pm 9 \\
76 \pm 12 \\
69 \pm 7 \\
60 \pm 10 \\
58 \pm 6 \\
<67\end{array}$ & $\begin{array}{l}50 \pm 15 \\
35 \\
60 \pm 10\end{array}$ & $\begin{array}{l}\text { ID (5) } \\
\text { EN (5) } \\
\text { EN (19) } \\
\text { EN (19) } \\
\text { ID (20) }\left(^{*}\right) \\
\text { FD (21) } \\
\text { DR (22) } \\
\text { ID (22) } \\
\text { ID (23) } \\
\text { FD (24) }\end{array}$ & 46 & 2.2 & 1.8 & 0 \\
\hline $\mathrm{Ge}$ & $\begin{array}{r}60 \pm 8 \\
67 \pm 13 \\
60 \pm 8 \\
73 \pm 9 \\
78 \pm 16 \\
100 \pm 10 \\
75 \pm 10 \\
78 \pm 7 \\
<82 \\
74 \pm 14\end{array}$ & $\begin{array}{r}30 \\
100\end{array}$ & $\begin{array}{l}\text { ID (5) } \\
\text { ID. (25) } \\
\text { ID (20) } \\
\text { ID (26) } \\
\text { ID (27) } \\
\text { HREM (27) } \\
\text { ID (23) } \\
\text { FD HREM } \\
\text { ID (24) } \\
\text { ID (24) }\end{array}$ & 65 & 3.3 & 2.9 & 0 \\
\hline $\mathrm{C}$ & $\begin{array}{l}279 \pm 41 \\
295 \pm 30\end{array}$ & & $\begin{array}{l}\text { FD (28) } \\
\text { EN (28) }\end{array}$ & 96 & 2.1 & 0.7 & 0 \\
\hline $\mathrm{GaSb}$ & $53 \pm 7$ & & ID (6) & 53 & 3.5 & 4 & 0.261 \\
\hline GaAs & $\begin{array}{ll}55 \pm & 5 \\
48 \pm & 6 \\
50 \pm 7 \\
41 \pm 7\end{array}$ & & $\begin{array}{r}\text { ID (6) } \\
\text { ID (29) } \\
\quad(30) \\
(30)\end{array}$ & 43 & 2.2 & 3.9 & 0.310 \\
\hline $\operatorname{InSb}$ & $38 \pm 4$ & & ID (6) & 43 & 3.3 & 3.8 & 0.321 \\
\hline $\mathrm{GaP}$ & $41 \pm 4$ & & ID (6) & 33 & 1.8 & 1.5 & 0.327 \\
\hline InAs & $30 \pm 3$ & & ID (6) & 30 & 2.2 & 2.2 & 0.357 \\
\hline $\operatorname{InP}$ & $18 \pm 3$ & & ID (6) & 17 & 1.2 & 1.1 & 0.421 \\
\hline
\end{tabular}

$\gamma_{\mathrm{i}} \quad$ : intrinsic stacking fault energy in $\mathrm{mJ} / \mathrm{m}^{2}$.

$\gamma_{\mathrm{e}} \quad$ : extrinsic stacking fault energy in $\mathrm{mJ} / \mathrm{m}^{2}$.

$\gamma_{i}^{\prime} \quad$ : reduced intrinsic stacking fault energy (see text for definition).

$\gamma_{\mathrm{i}} / \mu b$ : intrinsic stacking fault energy in $\mu b$ units.

$\gamma_{\mathrm{i}}^{\prime} / \mu b$ : reduced intrinsic stacking fault energy in $\mu b$ units.

$f_{\mathbf{i}} \quad$ : ionicity factor.

Notations for experimental techniques are in the text.

(*) Anomalous screw dislocation splitting related to dislocation point defects interaction (see Sect. 4.1). 
segments ; $v$ is proportional to the mean free path of kinks, $X$ :

$$
\begin{aligned}
& v=h X J \\
& v=a \tau b h^{2} / k T \nu_{0} \exp -\left(F_{\mathrm{k}}+W_{\mathrm{M}}\right) / k T
\end{aligned}
$$

where $a$ is the translation period along the dislocation.

Remarks : (i) complications due to the dissociated nature of dislocations have been neglected here and will be discussed in section 5.2.1.

(ii) $W_{\mathrm{M}}$ and $2 F_{\mathrm{k}}$ contain entropy terms which would contribute to pre-exponential factors in an Arrhenius plot. Marklund [36] has recently attempted to calculate these entropy terms.

The two above expressions differ in the activation energy for dislocation velocity. The transition between the two regimes seems to have been experimentally observed by Louchet [37] and Hirsch et al. [38]. Using in situ straining experiments in a high voltage microscope on $\mathrm{Si}\left(520^{\circ} \mathrm{C}<T_{\mathrm{C}}<650^{\circ} \mathrm{C}\right)$ Louchet [37] was able to evidence long perfect dislocation segments gliding with a velocity independent of their length whereas the velocity of short dislocation segments increased with dislocation length. The critical length $L_{\mathrm{c}}(=0.4 \mu \mathrm{m})$ for $\tau=$ $90 \mathrm{MPa}$ was interpreted as twice the mean free path of kinks so that an average velocity of kinks $v_{\mathrm{K}}$ can be deduced from dislocation velocity measurements. The same type of experiments were perfor-

in $1 \mathrm{~s} \mathrm{u}$ ying $\mathrm{t}$ e relaxation of widely dissociated dislocations. Partials moved under the back stress of the stacking fault $\left(\tau_{\mathrm{c}}=\right.$ $270 \mathrm{MPa})$ at temperatures between $200{ }^{\circ} \mathrm{C}$ and $450{ }^{\circ} \mathrm{C}$. Analysis of the velocity of $90^{\circ}$ partials led to the same order of magnitude for $L_{\mathrm{c}}$ than for the perfect dislocations (ie $\sim 0.2 \mu \mathrm{m}$ ).

The kink migration energy $W_{\mathrm{M}}$ estimated from these experiments

$$
\left(W_{\mathrm{M}}=k T \ln \left(\nu_{0} 2 h b a^{2} / k T\right) / v_{\mathrm{K}}\right)
$$

is $1.2 \mathrm{eV}$ which is about half the total energy for dislocation glide $\left(F_{\mathrm{K}} \sim 1 \mathrm{eV}\right)$. The kink migration energy can be determined either by the secondary Peierls potential or by overcoming weak obstacles along the dislocation [35, 39, 40]. However if the obstacle theory applies, the kinks would travel distance $l$ between every successful activation at obstacles ( $l$ distance between obstacles). Since it is expected that $l>a, W_{M}^{\prime}$ would be larger than $\mathrm{W}_{\mathrm{M}}$ deduced for an intrinsic kink migration mechanism $\left(W_{M}^{\prime}-W_{M}=2 k T \ln (l / a)\right)$, giving unreasonable values unless a large density of obstacles is assumed for which there is no explanation. Furthermore Hirth and Lothe [35] theory gives a better agreement with dislocation velocity measurements [41].

No experiments in CSC are presently available which could give informations about the magnitude of the kink migration energy. A length dependence of dislocation velocity has been reported in InSb [42] but the critical length $L_{\mathrm{c}}$ could not be determined.

The existence of this high secondary Peierls potential in ESC proves that the migration of kinks is as difficult as their nucleation contradictorily to fcc metals where even the primary Peierls potential is low [43]. This can be understood through the microscopic aspect of dislocation cores in SC: kink migration or nucleation require covalent bonds breaking. Kink migration is so difficult because partial dislocations and kinks are reconstructed.

Kinks and partial dislocation core configurations have been mainly discussed on their abilities to describe experimentals electronic levels measurements and the influence of doping (see § 5.2.1). Hirsch assumed that kinks can be either reconstructed or associated with dangling bonds [44, 45]. Examples of unreconstructed kinks are given in figure 7. A reconstructed kink is expected to have relatively low energy, but a high migration energy. In this scheme each double kink would have a dangling bond and the first step of kink nucleation would require a high energy. Jones [46] proposed a model in which all the kinks are reconstructed: dangling bonds exist only in the saddle point structure. However it needs two bonds to be broken at the saddle point and the saddle structure levels are not in agreement with experimental level

o e ca cu ation of Heggie and Jones [47].

To solve these contradictions, reconstruction defects along the core of the partials were taken into

a

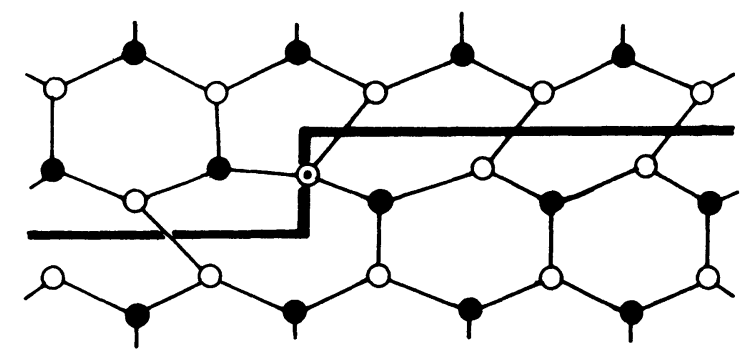

b

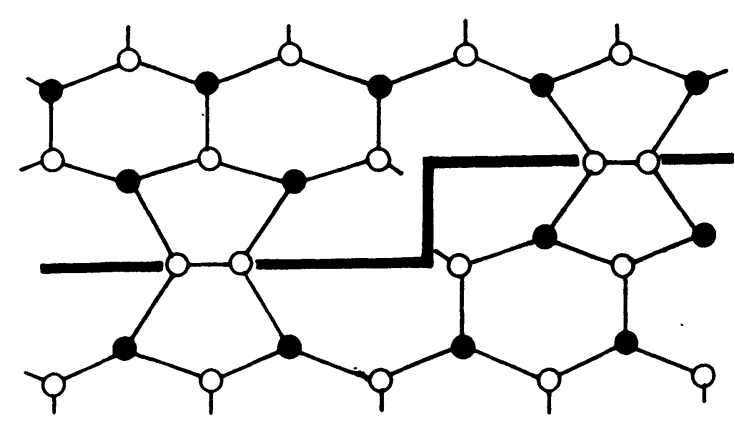

Fig. 7. - Example of unreconstructed kinks on reconstructed partial dislocations. In CSC open and filled circles are of different nature A or B. For $\alpha$ and $\beta$ partials, reconstructions occur between B-B and A-A atoms respectively $(\alpha=\mathrm{B}(g), \beta=\mathrm{A}(g))$. (a). $-90^{\circ}$ partial. (b). $-30^{\circ}$ partial. 
account. Along the core any particular atom has the choice of bonding with one or other neighbour in equivalent positions leading to relative displacements. When the bonding changes from one set of neighbours to another set of neighbours along the core an antiphase defect (APD) occurs associated with a dangling bond. This defect has been called «soliton » by Heggie and Jones [41, 47, 48] (Fig. 6c). (Solitons are most likely to form on $90^{\circ}$ partial and less likely on $30^{\circ}$ as this is the order of difficulty for reconstruction on partial dislocations [47].) These authors separated the dangling bond from the reconstructed kinks and assumed that solitons can move very easily along the dislocation line, even through reconstructed kinks. Each soliton can also create a double kink and facilitate the migration. Through this mechanism it can be understood that the nucleation and migration energies are quite similar.

Assuming that the length independent regime is established (see above), two cases can be considered following the relative values of the binding energy kink soliton $E_{\mathrm{KS}}$ and $k T$ [47]. This yields two different physical interpretations of the formation $F_{\mathrm{K}}$ and migration energy $W_{M}$ of the kinks :

- $E_{\mathrm{KS}}>k T$ : The experimental values $F_{\mathrm{K}}$ and $W_{\mathrm{M}}$ relate to the formation of a kink soliton complex on a reconstructed partial and its migration energy.

- $E_{\mathrm{KS}}<k T$ : The experimental values $F_{\mathrm{K}}$ and $W_{\mathrm{M}}$ relate to the formation of a reconstructed kink and its migration through a kink soliton saddle point and the dislocation velocity is proportional to the soliton density $\rho_{\mathrm{s}}$ :

$$
v=\tau b h^{2} a \nu_{0} \rho_{\mathrm{s}} / k T \exp -\left(F_{\mathrm{K}}+W_{\mathrm{M}}\right) / k T
$$

$\rho_{\mathrm{s}}$ is the probability a site being occupied by a soliton which can be expressed as $\rho_{\mathrm{s}} \alpha(1 / b) \exp \left(-F_{\mathrm{s}} / k T\right)$ at thermal equilibrium.

3.2 CSC. - This above analysis can be formally applied to CSC in the hypothesis where dislocations are reconstructed, which becomes easier as the covalent character of the bond increases (see $\$ 2.1$ ). Specific geometrical properties of dislocations in CSC related to dislocation velocities have been analysed assuming that kinks on partial dislocations can be considered as small dislocation segments which have $\alpha$ or $\beta$ character following the orientation of the partial (Fig. 8). Lateral kinks $\alpha$ or $\beta$ are expected to migrate with different velocities in relation to the respective mobilities of $\alpha$ and $\beta$ dislocations [49]. If this analysis can be relevant for «super " kinks which behave as usual dislocation segments, it seems unreasonable to equate a kink of one Burgers vector high with a dislocation segment whose specific properties are already related to kink migration. If $\alpha$ kinks are more mobile that $\beta$ kinks a

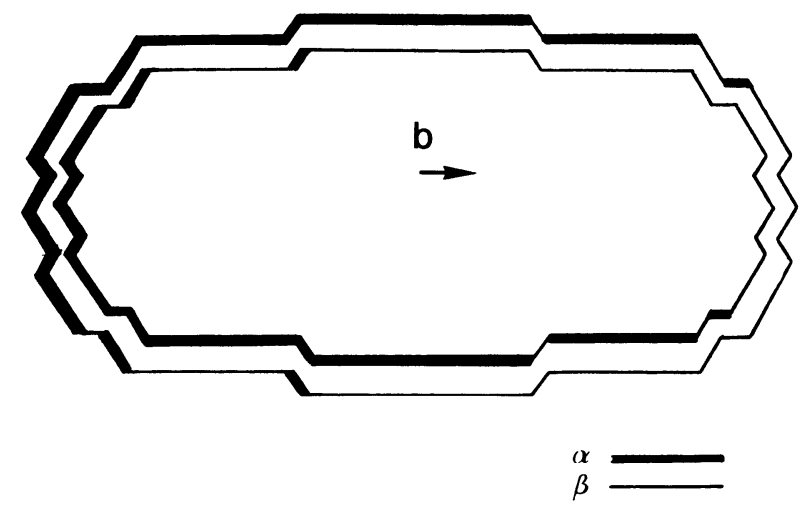

Fig. 8. - Glide loop in a CSC with the nature of super kinks on each of the partial dislocations.

screw dislocation will lose its rigid character (see § 5) by a rapid accumulation of the $\alpha$ kinks in the direction of the propagation of the rapid $\alpha$ front [50]. Furthermore this model cannot explain the asymmetry in the mobility of the two screw segments of a glide loop [51, 52].

\section{Dislocation point defects interactions.}

\section{1 «INTRINSIC » POINT DEFECTS.}

4.1.1 ESC. - Numerous spectroscopic and electrical experiments, depending on the deformation temperature $T$, have been interpreted in $\mathrm{Si}$ and $\mathrm{Ge}$ assuming that point defect clouds surround dislocations in plastically deformed materials. In Ge and Si changes in DLTS and Hall spectra obtained on crystals deformed below $0.6 T_{\mathrm{m}}$ can be recovered by annealing the crystals at higher temperatures. This has been explained in terms of disturbance of point defect equilibria [53], structural changes in the dislocation core [54] or defect cloud surrounding or left behind the dislocations $[55,56]$. However it is always difficult distinguishing between signals coming from dislocations themselves and point defects created during deformation since the two entities are created during the same test. Point defects can be induced by plastic deformation : dipole formation and break up, dislocation crossing, jog dragging... Although these metallurgical effects can explain the spectroscopic and electrical data, it is fashionable in ESC to relate the interpretations to the fact that dislocations can be either in the shuffle set or in the glide set : moving from one set to another is achieved by local climb i.e. point defect absorption or emission. This has explained the success of the concept of dislocation point defect association (see 1.1). It is not possible to decide on the validity of this model but some studies do show an effect of annealing and temperature deformation on the fine structure of dislocations i.e. dislocation splitting. Gomez et al. [20] studied in detail dislocation splitting in $\mathrm{Ge}$ and Si. Specimens 
were prepared by bending $\langle 111\rangle$ slices at high temperature $\left(600^{\circ} \mathrm{C}\right.$ and $800{ }^{\circ} \mathrm{C}$ for $\mathrm{Ge}, 880^{\circ} \mathrm{C}$ for $\mathrm{Si})$. After deformation the slices were annealed at the deformation temperature for $10 \mathrm{~min}$ and then slowly cooled. Two sets of dissociation widths were found for screw and near screw segments in both $\mathrm{Si}$ and Ge. The narrow dissociation which was in accordance with the measured « normal " stacking fault energy on edge segments was found to be associated with an intrinsic stacking fault, whereas large dissociation was found to be associated with an extrinsic nature of the fault. A similar feature was found by Packeiser and Haasen [26] on Ge deformed at $600{ }^{\circ} \mathrm{C}$ and $400{ }^{\circ} \mathrm{C}$ and annealed at $450{ }^{\circ} \mathrm{C}$. No identification of the stacking fault nature was made but constrictions were found to separate two screw dislocation segments with the same splitting width. Constrictions were found to be intrinsic to the nature of the dislocation since their concentration was found to be greater than impurity concentrations. Aristov et al. [57] found the two sets of screw dislocation dissociation in $\mathrm{Si}$ deformed at $680^{\circ} \mathrm{C}$ without annealing, and in the same samples annealed at $500{ }^{\circ} \mathrm{C}$ for $1.5 \mathrm{~h}$. The splitting was only « normal » after annealing the sample at $790^{\circ} \mathrm{C}$ for $15 \mathrm{~min}$. These dislocation splitting measurements were correlated with DLTS spectra.

Several explanations for these anomalous splitting widths on screw and near screw dislocations were ro os

basis of extrinsic fault nucleation at constrictions or through the nucleation of small prismatic loops of point defects reacting with the dislocation. This requires that $\gamma_{\mathrm{e}}=0.5 \gamma_{\mathrm{i}}$ which is not realistic (see Tab. II). An alternative explanation [58] was that partials are sufficiently charged so that an additional repulsive term between the two partials can be important. Impurities on dislocations can induce the nucleation of an extrinsic stacking fault and give rise to this additional Coulomb interaction between the partial dislocations.

This anomalous splitting has not yet received a satisfactory explanation.

4.1.2 CSC. - CSC dislocation point defect interactions are complicated with respect to ESC by the presence of two sublattices. During dislocation climb the compound nature of the lattice requires that two atomic species, instead of one, be absorbed or emitted to produce jog motion and dislocation climb. If climb proceeds by the simultaneous addition of the two types of atoms an $\mathrm{A}(\mathrm{g})$ core will remain $\mathbf{A}(\mathrm{g})$ in character. If however climb involves two discrete stages : addition of one row of $B$ atoms followed by one row of $A$ atoms, the $A(g)$ character of the dislocation will alternate between $B(s)$ and $A(g)$, configurations. These two types of mechanisms involve two different types of jog : the first one

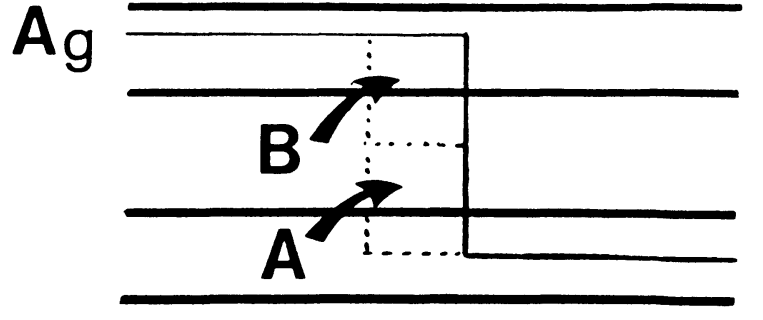

a
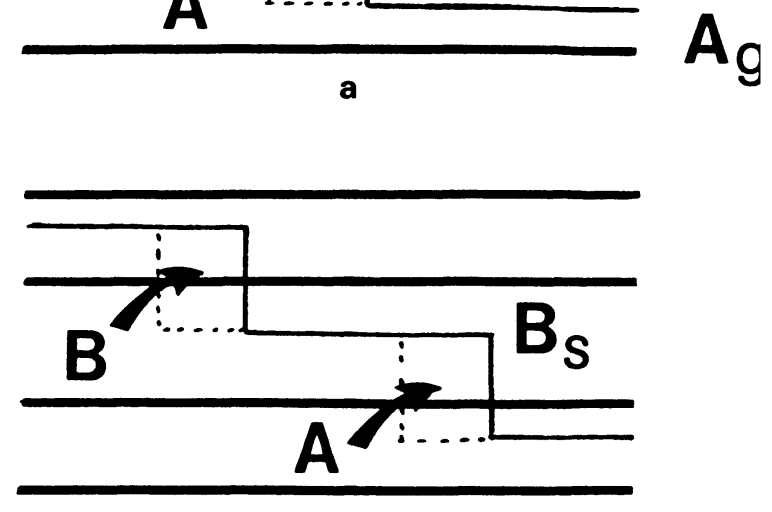

b

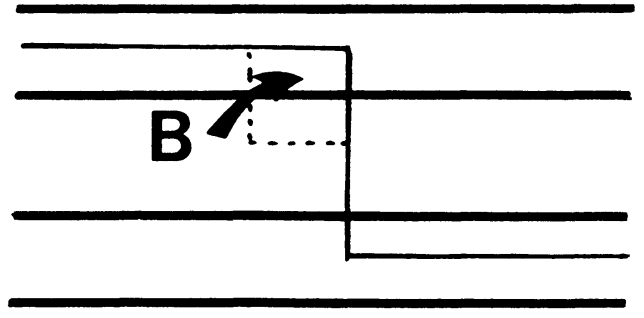

Fig. 9. - Scheme for the absorption of the two atomic species at jogs in CSC (only one extra half-plane is shown). (a). - Absorption of the two atomic species at a double jog. (b). - Absorption of each of the two atomic species at two single jogs. (c). - Dissociation of a double jog $(A, B)$ into two single jogs $A, B$.

a «double » jog A, B, the second one two single jogs A or B (cf. Fig. 9). Note that a double jog can be dissociated into two single jogs $A, B$ by absorption of one atom (A or B) (Fig. 9c). These different climb mechanisms depend on the coupling of the two different species insuring the flux of matter in the diffusion process. The coupling of the flux of the defects of the two sublattices is not as necessary as it is in ionic crystals when coulombic effects induce a Nernst field which equalises the migration velocity of the two species [59]. The coupling of these two fluxes should depend on the ionic character of the compound and of the state of charge of the two diffusing species. In any case an asymmetry exists between $\mathrm{A}$ and $\mathrm{B}$ dislocations climb process. This asymmetry has been evidenced for dipole climb in degraded gallium arsenide lasers [60].

It is, furthermore, rather unlikely that point defects of the two species are always present in equal supersaturations. Petroff [61] explained low tem- 
perature climb observations in GaAs based laser devices by the migration of one type of point defect $\mathrm{Ga}_{i}$ to the dislocations. $\mathrm{A} \mathrm{Ga}_{i}$ atom moving to the dislocation is incorporated to the extra half- plane whereas a vacancy $V_{A s}$ is emitted from the dislocation to restore a stoichiometric $\mathrm{As}_{\mathrm{As}} \mathrm{Ga}_{\mathrm{Ga}}$ couple at the dislocation core (Fig. 10). To explain small intrinsic dislocation loops appearing as by-product of the climb mechanism he assumed that a reaction $\mathrm{V}_{\mathrm{AS}}+\mathrm{Ga}_{\mathrm{Ga}} \rightarrow \mathrm{Ga}_{\mathrm{As}}+\mathrm{V}_{\mathrm{Ga}}$ restores a $\mathrm{V}_{\mathrm{Ga}}$ vacancy in the $V_{A s}$ rich region together with an antisite $\mathrm{Ga}_{\mathrm{As}}$ so that stoichiometric vacancy loops $\mathrm{V}_{\mathrm{Ga}}+$ $\mathrm{V}_{\mathrm{As}}$ can be formed. The driving force for the climb mechanism is the reaction $\mathrm{Ga}_{i} \rightarrow \mathrm{V}_{\mathrm{As}}$ (at the dislocation) which requires the chemical potential of $\mathrm{V}_{\mathrm{As}}$ to be smaller than that of $\mathrm{Ga}_{i}$. It is clear from this example that dislocations act as moderators of the population of non stoichiometry defects.
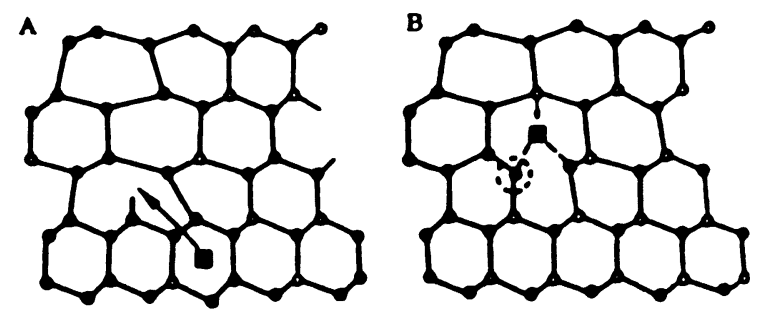

C

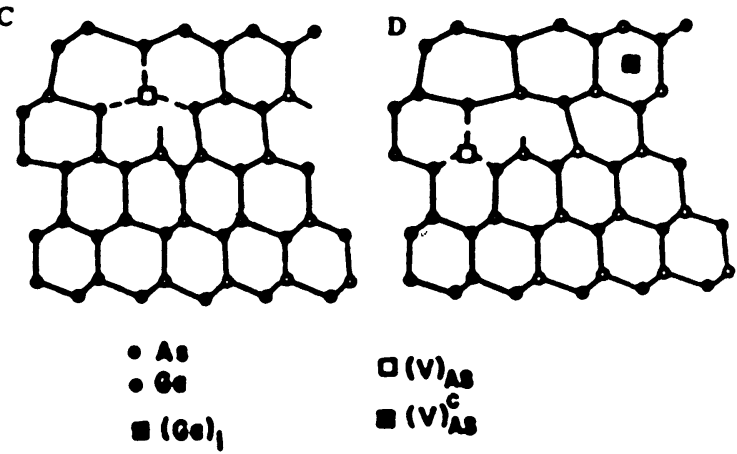

Fig. 10. - Dislocation climb model in CSC for a supersaturation of defects on one type of the two sublattices (after Petroff [6]).

An important native defect in GaAs is the $\mathrm{EL}_{2}$ centre. The concentration of $\mathrm{EL}_{2}$ centres has been found to be correlated with the dislocation density in GaAs [62]. $\mathrm{EL}_{2}$ was thought to be $\mathrm{As}_{\mathrm{Ga}}$ but it appeared to be a complex which contains $\mathrm{As}_{\mathrm{Ga}}$ [63]. Models to relate $\mathrm{EL}_{2}$ to dislocation density rely on dislocation climb [62] or dislocation glide of one type of dislocation [64] : incorporation of a defect supersaturation $\mathrm{As}_{i}$, in the climbing or gliding dislocation, restores $\mathrm{As}_{\mathrm{Ga}}$ from energetic considerations relative to point defects. However this requires a supersaturation of one type of point defect whose concentration is given at the beginning of the deformation test. It seems more likely to attribute the presence of $\mathrm{As}_{\mathrm{Ga}}$ to the creation of
$\mathrm{V}_{\mathrm{As}}$ and $V_{\mathrm{Ga}}$ either by climb for high temperature deformation or by dislocation dipole formation at lower temperatures [65], these defects being unstable through the reaction $\mathrm{V}_{\mathrm{Ga}}+\mathrm{As} \rightarrow \mathrm{As}_{\mathrm{Ga}}+\mathrm{V}_{\mathrm{As}}$.

Observations of dislocation splitting in GaAs and other III-V compounds have not yet reported the anomaly of dislocation splitting on screw dislocations as a function of deformation temperature or/and annealing temperature.

4.2 EXTRINSIC POINT DEFECTS. - One can distinguish between two types of effects for the interaction of dislocations with extrinsic point defects in SC: electronic effects for electrically active impurities (i.e. associated with Fermi Level displacement) and classical metallurgical effects for any type of impurities (i.e. direct interactions of dislocations with point defects). Electrically active impurities can affect the dislocation mobilities in these two ways following temperature, stress and strain rate ranges.

4.2.1 Electronic effects. - It has been shown that respectively III or V elements in ESC can affect the mobilities of dislocations the same way whatever their chemical nature, or size effect in the matrix can be. They act respectively as acceptor or donor in these crystals and change the Fermi level in the band gap $\left(E_{\mathrm{F}}=k T \ln \left(C_{\mathrm{v}} T^{3 / 2} / N_{\mathrm{c}}\right)\right.$ in the case of acceptors). The same effect is also found in CSC with respectively II or VI elements (for IV elements the effect ( $n$ or $p$ ) depends on their position in the lattice sites, replacing III or V elements).

Two classes of theory explain the electronic effect on dislocation mobilities :

- The line charge of a dislocation in a SC depends solely on the position of the Fermi level relative to that $E_{0}$ of the dislocation which depends on the dislocation character. Haasen [66, 67] used this dislocation charge concept. A charged dislocation is unstable versus double kink nucleation : the formation energy of a double kink is reduced by the dislocation charge since it promotes separation of repulsive charges. He obtains for the decrease of the double kink formation energy on a dislocation with a charge $Q$ per unit length :

$$
\Delta 2 F_{\mathrm{k}}=-\frac{2 Q^{2} a^{2}}{\varepsilon} \exp (-a / 2 \lambda)
$$

where $e$ is the elemental charge, $\varepsilon$ the dielectric constant, $a$ the distance between two nearest Peierls valleys, $\lambda$ the Debye screening length. The dislocation charge can be expressed as :

$$
Q=-e f / b
$$

where $f$, the ratio of charged dislocation sites can be determined from the Fermi energy :

$$
E_{\mathrm{F}}=E_{0}+2 e^{2} f / \varepsilon b\left(\ln \lambda / r_{0}+1 / 2\right)-T S .
$$


However the predicted effect has not the right order of magnitude [61].

Hirsch $[44,45,68]$ considered that the formation of a double kink leads to the generation of acceptor $E_{\mathrm{Ka}}$ or donor $E_{\mathrm{Kd}}$ levels in the band gap. Charged as well as neutral kinks can then exist, the concentration of charged kinks depending on the position of the Fermi level. It is assumed that the concentration $C_{\mathrm{k}}$ of neutral kinks is constant so that the total concentration of kinks increases. Since the dislocation velocity depends on the concentration of kinks and their mobility, the velocity will depend on the position of the Fermi level partly because of the effect on the concentration of charged kinks and partly because the velocity of the kink may be charge dependent. The concentrations of positively charged $C_{\mathrm{k}}^{+}$(or negatively charged : $C_{\mathrm{k}}^{-}$) is expressed as :

$$
\begin{aligned}
& C_{\mathrm{k}}^{+} / C_{\mathrm{k}}^{0}=\exp \left(E_{\mathrm{Kd}}-E_{\mathrm{F}}+e V\right) / k T ; \\
& C_{\mathrm{k}}^{-} / C_{\mathrm{k}}^{0}=\exp \left(E_{\mathrm{F}}-E_{\mathrm{Ka}}-e V\right) / k T
\end{aligned}
$$

where $\mathrm{eV}$ is the electrostatic energy of the charged dislocation line including the charge of the kinks. If the dislocation velocity is controlled by positively charged kinks or negatively charged kinks, its velocity is respectively $v^{+}$and $v^{-}$, which can be compared to its velocity $v_{0}$ when controlled by neutral kinks.

$$
\begin{aligned}
& v^{+} / v_{0}=\exp \left(E_{\mathrm{Kd}}-E+e V-W_{\mathrm{M}}^{+}\right) / k T \\
& v^{-} / v_{0}=\exp \left(E_{\mathrm{F}}-E_{\mathrm{Ka}}-e V-W_{\mathrm{M}}^{-}\right) / k T
\end{aligned}
$$

$W_{\mathrm{M}}^{+}$and $W_{\mathrm{M}}^{-}$are the differences in activation energies of migration for positively and negatively charged kinks and neutral kinks.

The total dislocation velocity due to charged and uncharged kinks is $v_{0}+v^{+}$or $v_{0}+v^{-}$; since the concentration of neutral kinks is constant, the dislocation velocity will increase with increasing concentrations of charged kinks. If the kinks' levels are near the middle of the gap the velocities of dislocations of $n$ and $p$ type materials should be greater than that of intrinsic material, i.e. the strength of the crystal should be a maximum for intrinsic material ; doping by electrically active impurities causes softening. On the other hand, if the kinks' levels are close to the valence band the velocity should decrease from $\mathrm{n}$ to $\mathrm{p}$ type material, i.e. $\mathrm{n}$ type material is weaker and $p$ type stronger than intrinsic material. In this case doping of the pure material can cause hardening or softening depending on the impurity. These behaviours are, respectively, the cases of $\mathrm{Si}$ and $\mathrm{Ge}$. However it is difficult to establish a link between the level obtained experimentally and the kinks' levels. Furthermore levels at the saddle point $E_{\mathrm{Sa}}$ and $E_{\mathrm{Sd}}$ may be the relevant levels controlling the dislocation velocity since $W_{\mathrm{M}}=E_{\mathrm{Sa}}-E_{\mathrm{Ka}}$, $W_{\mathrm{M}}=E_{\mathrm{Kd}}-E_{\mathrm{Sd}}$ if the electrons follow the motion of ions during the activation to the saddle point (adiabatic principle) [45, 68].

This theory applies strictly to the low stress and high temperature regime, where double kink nucleation is not important and kink concentration is given by thermodynamic considerations [35]. However the theory remains virtually the same under the adiabatic principle for high stresses and low temperature when dislocation velocity is controlled by the nucleation of double kinks.

The other models yield formally the same expression for dislocation velocity. In the strainedbond model of Jones [46] the effect of doping is ascribed to charged saddle point configurations. Kinks are neutral and thermodynamical equilibrium is established with the electron gas at the saddle point. In the soliton-model the relevant levels are those of the saddle point for kink-soliton motion [47, 48].

4.2.2 Metallurgical effects. - In contrast to electronic effects which are unlocalized, metallurgical effects come from localized interactions between dislocation impurities (substitutional or interstitial). This results, most of the time, in hardening effects which implies that these point defects are obstacles spread out in the dislocation glide plane. Point defects can also build clusters which interact with dislocations : this case is not taken into account in this part of the paper.

Point obstacles are immobile at low temperature or can diffuse to the dislocation at higher temperature. One can distinguish among the theoretical models related to dislocation point obstacle interaction those which rely upon short distance interaction, which can be overcome by thermal activation, and those which rely on long distance interaction of athermal type. In what follows only short distance interactions are taken into account.

In metals elastic interactions are the only relevant interactions and can be of two types : size effect $\left(E_{\mathrm{s}}\right)$, modulus effect or inhomogeneity interaction $\left(E_{\mathrm{I}}\right)$ [35]. The size effect arises from the hydrostatic component of the stress tensor around an edge dislocation and the inhomogeneity interaction is related to the hard or soft character of the defect with respect to the elastic constants of the matrix. This can be formally expressed by :

$$
E_{\mathrm{int}}^{\mathrm{e}}=E_{\mathrm{s}}+E_{\mathrm{I}}
$$

with

$$
\begin{aligned}
& E_{\mathrm{S}}=-P_{i j} \varepsilon_{i j}\left(r_{\mathrm{s}}\right) \\
& E_{\mathrm{I}}=-1 / 2 \varepsilon_{i j}\left(r_{\mathrm{s}}\right) \alpha_{i j k l} \varepsilon_{k l}\left(r_{\mathrm{s}}\right)
\end{aligned}
$$

where $P_{i j}$ is the dipole tensor of the defect and $\varepsilon_{i j}$ is the strain induced by the dislocation at point $r_{s} \cdot \alpha_{i j k l}$ is the dielastic polarizability which contributes to the change in elastic constants of crystals to which point defects have been added [69]. 
In this formalism is included the usual size effect for isotropic defects in which $P_{i j}=\Delta V$ ( $\Delta V$ volume of formation of the point defects) and dipolar interaction when this relaxation volume is anisotropic and creates an asymmetric distorsion in the lattice. This latter effect explains for example the hardening effect of $\mathrm{C}$ in $\mathrm{Fe}[70]$ and the hardening of ionic crystals [71].

In SC an additional effect has to be taken into account : the electrostatic interaction between dislocations and point defects. The strength of this interaction depends on the state of charge of the point defects and the dislocation. For dissociated dislocations all these interactions have to be summed up on the different partials. Furthermore dislocation-impurity reactions can modify the core structure of dislocations and consequently their movement at an atomic scale [72].

At higher temperatures impurities are not immobile any more and can diffuse to the dislocation. An impurity atmosphere is built around the dislocation (Cottrell atmosphere or Snoeck atmosphere depending on the substitutional or interstitial nature of the impurity). Impurities can diffuse along the dislocation core by pipe diffusion mechanisms. Dynamic effects occur which are related to the respective motion of dislocation and impurities: impurity dragging, locking of the dislocation or break away from the impurity atmosphere. The point defect diffusion coefficient and its binding energy with the dislocation are the relevant quantities to analyse these mechanisms.

The analysis of the various possible mechanisms is outside of the scope of this paper and the reader is referred for example to Hirth and Lothe [35] for a review of these mechanisms. However the work of Brion et al. [73] on highly doped Ge can be given as an example. Figure 11 shows the dislocation velocity as a function of $T$. The parts $\mathrm{A}$ and $\mathrm{B}$ are attributed

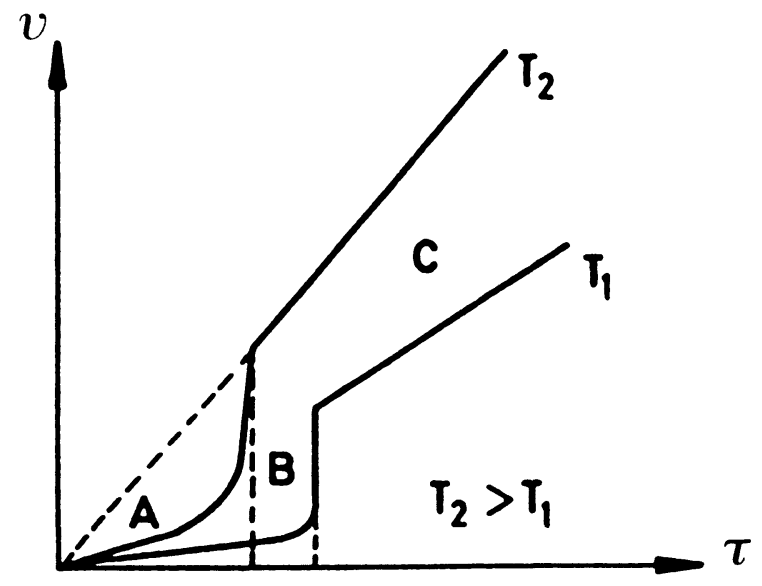

Fig. 11. - Dislocation velocity vs. stress in doped Ge. Regions $A$ and $B$ are explained by metallurgical effects (after Brion et al. [73]). to metallurgical effects. In region A (small $v$ ) it is possible for dislocations to drag their solute clouds with them steadily and the dislocation velocity can be expressed by :

$$
v=D_{\mathrm{s}} F / k T .
$$

Where $D_{\mathrm{s}}$ is the diffusion coefficient of the solute atom and $F$ the interaction force between dislocation and an atom it is dragging. In region $B$ a pinning effect is evidenced. The critical stress for dislocation unpinning is linear in $C$ (concentration of impurities) and directly related to the impurity-dislocation binding energy.

$$
\tau_{\mathrm{B}}=C k T / b^{3} \exp -\left(\alpha U_{0} / k T\right)
$$

In this region pinning depinning effects affect the macroscopic plasticity (see part II).

\section{Dislocation velocity measurements.}

The theory of dislocation glide in SC which has been reviewed in $\S 3$ was developed to rationalize a wealth of data on dislocation velocities. It must be stressed that in no other class of materials is the dislocation mobility known with a comparable accuracy. Glide velocities of dislocations in Si [74-76], $\mathrm{Ge}$ [77-79] and several III-V compounds (InSb [8082], GaAs [83-85], InAs, GaSb [86], InP [87-88]) have been measured by several groups, in some cases for more than twenty years [77-89], and general trends are experimentally well established, especially in ESC. However some difficulties remain : from the experimental point of view, firstly, whilst different authors are often in very close agreement, detailed investigations have shown that dislocation velocities may be very sensitive to parameters that are difficult to control and therefore often overlooked such as residual impurity content, non-shear component of the applied stress, thermal history of the crystals under study. Secondly, many features have not yet been given a really satisfying theoretical explanation and elementary rate controlling mechanisms are still a matter of controversy.

The present state of knowledge can be summarized as follows.

5.1 LOOP GEOMETRY. - As checked by in situ observations (X-ray topography [90-92] and HVEM [93-95]), dislocation loops retain crystallographic $\langle 110\rangle$ orientations during their glide in $\{111\}$ planes (see Sect. 1.1) (Fig. 12). It is quite sure that dislocations glide in the dissociated state (which is not a convincing proof that they belong to the glide set). Glide motion appears to be smooth at the TEM scale, in Si and Ge [93-94]. Only occasionnal pinning points are observed to locally retard the dislocations and result in transient acceleration 

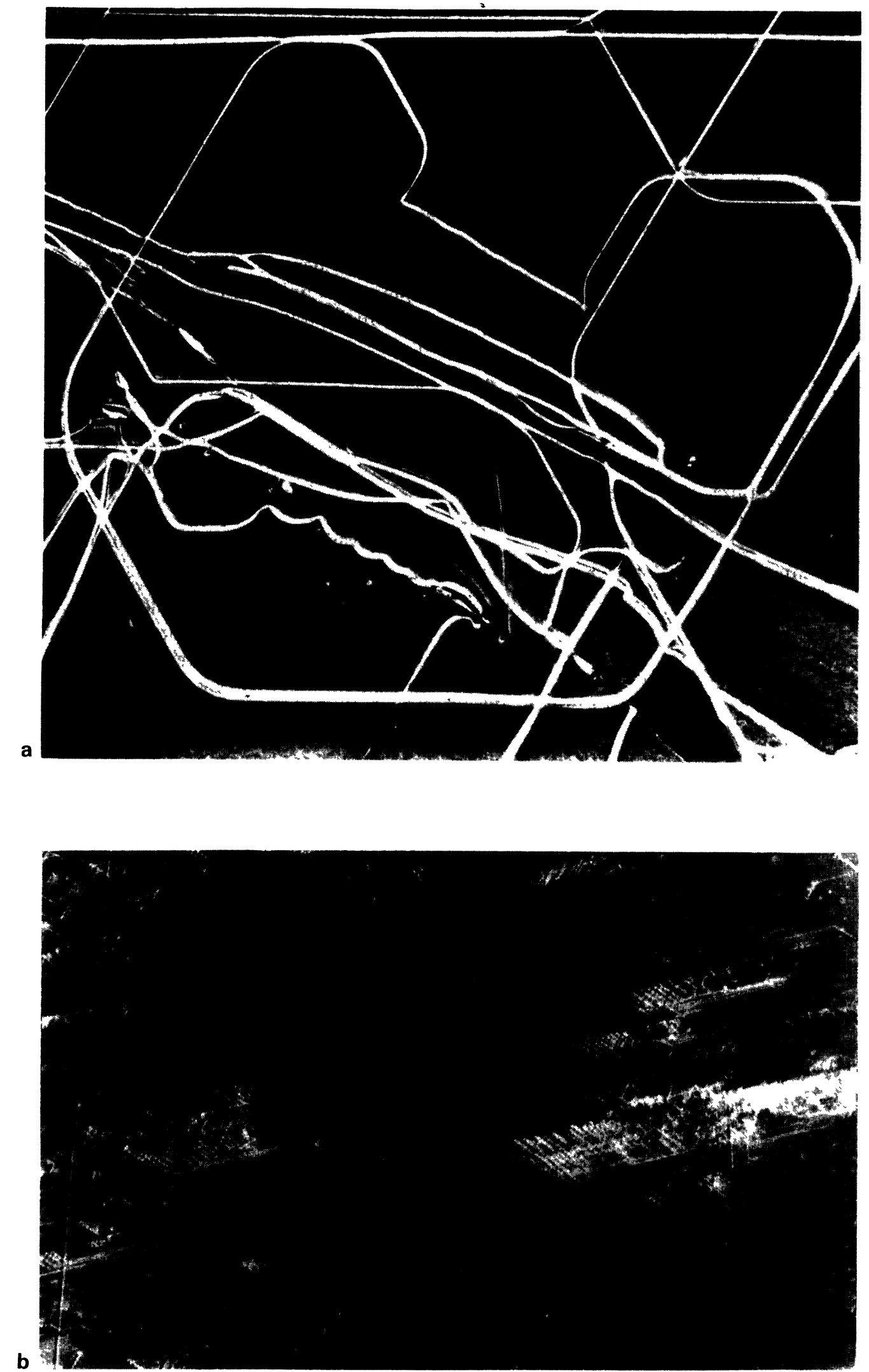

Fig. 12. - $\langle 110\rangle$ orientations of moving dislocation segments in semiconductors. (a). - Dislocation loop bulging out from a tangle formed during high temperature pre-strain. Silicon. TEM after cooling under load. $T=550^{\circ} \mathrm{C}$, $\dot{\gamma}=2 \times 10^{-5} \mathrm{~s}^{-1}, \tau=45 \mathrm{MPa}$. Test interrupted before the upper yield point $\left(\tau_{\mathrm{uy}}=72 \mathrm{MPa}\right)$. Marker : $1 \mu \mathrm{m}$. (R. Allem, unpublished). (b). - InP (S doped). X-Ray topograph. Marker : $500 \mu \mathrm{m}$. (Half-loops consist of $\beta$ and screw dislocations. In $\mathrm{S}$ doped InP, velocities of $\alpha, \beta$ and screw dislocations are of the same order of magnitude, a situation not typical of III-V compounds). 
when, after unpinning, cusps catch up the rest of the line.

The situation is less clear in compounds. Sato and Sumino [94] reported jerky motion in GaAs, but Caillard recently observed a very smooth motion in InSb [42].

\subsection{VELOCITIES OF LARGE DISLOCATION SEGMENTS} $(L \gg X)$ IN INTRINSIC MATERIALS. - In most eXperiments, velocities, measured by double etching or $\mathrm{X}$-ray topography, were averaged over travel distances of 10 to $100 \mu \mathrm{m}$, the initial diameter of (half-) loops being larger than $50 \mu \mathrm{m}$. In those conditions, velocities are not length dependent (see § 3.1).

The investigated range of temperature and resolved shear stress was long restricted to, typically,

$$
0.45 T_{\mathrm{m}}<T<0.65 T_{\mathrm{m}}
$$

( $T_{\mathrm{m}}$, absolute temperature of melting)

$5 \times 10^{-5} \mu<\tau<10^{-3} \mu \quad(\mu$, shear modulus).

This could be referred to as a «central " range of measurements for which data are available from different groups and exhibit, at least for $\mathrm{Si}$ and $\mathrm{Ge}, \mathrm{a}$ very good agreement (Fig. 13).

Recently, higher temperatures have been investigated and also higher stresses. For practical reasons, it is not possible to avoid some correlations between high temperature and low stress or conversely high stress and low temperature, in order to keep the resulting velocity in the measurable range. In the following an attempt has been made to separate what is due to stress from what is due to temperature.

5.2.1 Temperature dependence. - In the central range of measurements, the temperature dependence of the dislocation velocity is accurately described by an Arrhenius law :

$$
v \sim \exp (-Q(\tau) / k T) .
$$

The apparent activation energy, $Q$, is about 1 or $2 \mathrm{eV}$ for most SC. $Q$ values are listed in table III for non or slightly doped materials, i.e. electrically intrinsic at the temperature of measurements.

$Q$ is significantly stress dependent. $Q$ strongly increases below a certain stress of the order of 10 to $20 \mathrm{MPa}$, especially in Ge. (According to Imai and Sumino [76] the smaller increase of $Q$ in Si could be ascribed to residual impurities.) Above that stress, $Q$ is a weakly decreasing function of $\tau$. In $\mathrm{Si}$, $Q \cong 2.2 \mathrm{eV}$ at $\tau=20 \mathrm{MPa}, Q \cong 1.8 \mathrm{eV}$ at $\tau=$ $300 \mathrm{MPa}$.

$Q$ slightly depends on the character of the dislocation. In $\mathrm{Si}$ for example, the stress dependence is more marked for $60^{\circ}$ dislocations than for screws. In III-V compounds, $Q$ is consistently smaller for $\alpha$ dislocations than for $\beta$ and screws.

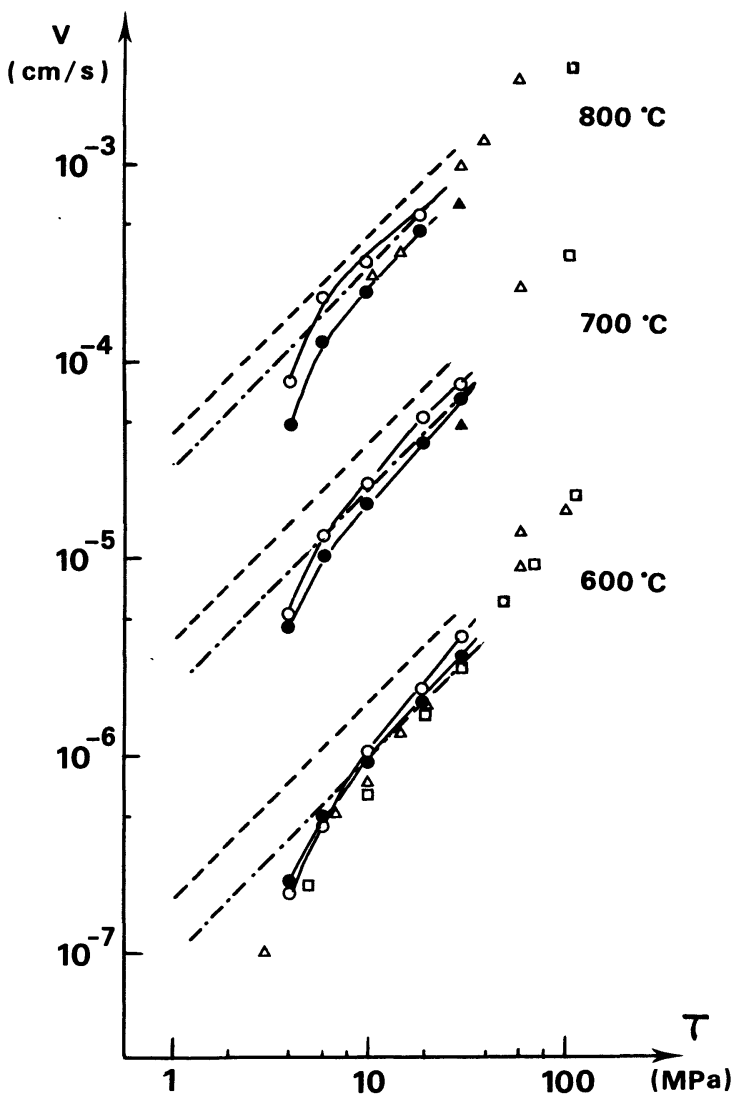

Fig. 13. - Dislocation velocities in intrinsic «pure» silicon. Comparison of data obtained by several groups in the « central » range of measurements

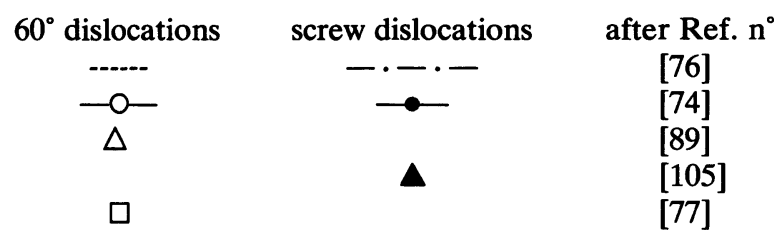

The Arrhenius law can break at some critical temperature as evidenced recently in $\mathrm{Si}$ and $\mathrm{Ge}$. Nikitenko and coworkers have measured $60^{\circ}$ dislocation velocities up to $0.9 T_{\mathrm{m}}$ in $\mathrm{Si}$ (p type: $4 \times$ $10^{13} \mathrm{~cm}^{-3}, 2 \mathrm{MPa} \leqslant \tau \leqslant 45 \mathrm{MPa}$ ) [95] and in Ge $(\rho>15 \Omega . c m, 1 \mathrm{MPa} \leqslant \tau \leqslant 30 \mathrm{MPa})$ [96]. In both cases a marked change in $v(T)$ was observed above $\sim 0.75 T_{\mathrm{m}}\left(600^{\circ} \mathrm{C}\right.$ for $\mathrm{Ge}, 1050^{\circ} \mathrm{C}$ for $\left.\mathrm{Si}\right)$. The velocity can still be described by an Arrhenius law, but both $Q$ and the prefactor are changed. In $\mathrm{Si}, Q$ increases from $2.2 \mathrm{eV}$ up to $4 \mathrm{eV}$, but this is compensated by a very large prefactor $\left(10^{12}\right.$ to $\left.10^{14} \mathrm{~cm} \cdot \mathrm{s}^{-1}\right)$ so that the resulting velocity is larger than extrapolated from lower temperatures. In $\mathrm{Ge}$, the situation is reversed. A lower, apparently stress independent, $Q \sim 1.8 \mathrm{eV}$ is found and the velocity is smaller than extrapolated from lower temperatures. Such changes have not received a clear explanation so far.

Correlation between $Q$ and physical quantities have been looked for, in an attempt to normalization to give an unified description of all semiconducting 
Table III. - Apparent activation energies, $Q$, for dislocation velocities in undoped element and compound semiconductors (selected references). (In some cases the quoted figures have been interpolated from $Q(\tau)$ curves given in original papers. Typical error bar is $\pm 0.1 \mathrm{eV}$.)

\begin{tabular}{|c|c|c|c|c|c|}
\hline Material & $Q$ screw $(\mathrm{eV})$ & \multicolumn{2}{|c|}{$Q 60^{\circ}(\mathrm{eV})$} & $\tau(\mathrm{MPa})$ & $T(\mathrm{~K})$ \\
\hline & 2.17 & \multicolumn{2}{|c|}{2.43} & 4 & $793-1073$ \\
\hline & 2.17 & \multicolumn{2}{|c|}{2.16} & 30 & $763-1023$ \\
\hline S1 & 2.35 & \multicolumn{2}{|c|}{2.20} & $1.2-40$ & $876-1046$ \\
\hline & - & \multicolumn{2}{|c|}{1.82} & 294 & $598-695$ \\
\hline Ge & 1.77 & \multicolumn{2}{|c|}{2.93} & 6 & $693-813$ \\
\hline ve & 1.45 & \multicolumn{2}{|c|}{1.63} & 20 & $653-793$ \\
\hline III-V & & \multicolumn{2}{|l|}{$Q_{\alpha}$} & & \\
\hline \multirow[t]{2}{*}{$\mathrm{GaSb}$} & - & 1.3 & 1.6 & 15 & $523-723$ \\
\hline & - & 0.95 & 1.3 & 30 & $\begin{array}{l}\sim 473-673(\alpha) \\
\sim 523-773(\beta)\end{array}$ \\
\hline \multirow[t]{5}{*}{ GaAs } & 1.1 & - & - & 25 & $523-723$ \\
\hline & - & 1.0 & 1.35 & 20 & $423-573(\alpha)$ \\
\hline & & & & & $573-773(\beta)$ \\
\hline & - & 0.78 & 1.1 & $4-13(\alpha)$ & $293-603(\alpha)$ \\
\hline & & & & $8(\beta)$ & $543-603(\beta)$ \\
\hline \multirow{2}{*}{$\mathrm{InSb}$} & - & 0.73 & 0.8 & 30 & $323-523(\alpha)$ \\
\hline & 1.1 & 0.88 & 1.2 & 5 & $\begin{array}{l}350-573(\beta) \\
323-438(\alpha)\end{array}$ \\
\hline$\ldots-$ & & & & & - \\
\hline InAs & - & 1.0 & 1.2 & 10 & $305-573$ \\
\hline
\end{tabular}

Réf. materials. Convincing correlations could not be established, probably because $Q$ is a complex quantity containing the kink migration energy $W_{\mathrm{M}}$, the kink pair formation energy $2 F_{\mathrm{k}}$ and, may be, also some characteristics of other defects (intrinsic reconstruction defects, binding energies of impurities with the dislocation core...). Let us mention the cơrrelation proposed by Gilman between $Q$ and $E_{\mathrm{g}}$, the band gap energy [97]. Indeed, for not too different ionicity values, the lower the band gap, the lower $Q$ (ex. : InSb vs GaAs).

5.2.2 Stress dependence. - The stress dependence of dislocation velocity is usually described by the empirical power law

$$
v \cong v_{0}\left(\tau / \tau_{0}\right)^{m}
$$

with a stress exponent between 1 and 2 . This description is better at high stresses (it was checked in $\mathrm{Si}$ from $\sim 30 \mathrm{MPa}$ to $\sim 300 \mathrm{MPa}$ ), than at low stress where the exponent can be not only temperature dependent but stress dependent i.e. the stress dependence $v(\tau)$ exhibits a bend on a double logarithmic plot, with higher «local $m \cong 4$ as observed in Ge [77-79].

Clearly such a description is not very satisfactory. There is little doubt that the stress dependence of $v$ for a good part results from the stress dependence of the activation energy but this has not been rationalized yet. In this respect it would be very important to measure the stress dependence of the kink migration energy $W_{M}$ and the kink pair formation energy $2 F_{\mathrm{k}}$. Estimation of $W_{\mathrm{M}}$ from TEM investigations can hardly be extended to a wide range of conditions. Very promising could be the pulse train technique of Nikitenko, Farber and Iunin [98], the principle of which well deserves a few words : the stress is applied in trains of short pulses of duration $t_{\mathrm{i}}$ separated by intervals of duration $t_{0}$. It is then possible to compare the average dislocation velocity resulting from the same total stress duration but for independently varying values of $t_{\mathrm{i}}$ and $t_{0}$. A large variety of results can be obtained for short pulses : pulses can be too short for any successful activation event to occur or a very limited expansion of a kink pair is only possible and back 
motion, more or less complete depending on $t_{0}$, can occur during the interval between two pulses. So far early results with this technique confirm the high value of $W_{M}$ in Si.

An important point concerning the stress dependence is that two different regimes must be distinguished, because of the dislocation dissociation : if the stress is low, a kink pair formed on one partial should not be stable against the restoring action of the stacking fault. For $\tau<\tau_{c}$, kink pairs must form in both partials in a correlated manner in order to promote a net motion of the dislocation line. At higher stresses, kink pair formation may occur separately on each partial. The critical stress, $\tau_{c}$, depends on the dissociation width i.e. on the screw or $60^{\circ}$ character of the dislocation :

$$
\tau_{\mathrm{c}} \cong \frac{\mu a b_{\mathrm{p}}}{4 \pi d_{0}^{2}} \cdot \beta=\frac{\pi}{\beta} \frac{\gamma^{2} a}{\mu b_{p}^{3}}
$$

where $a$ is the distance between Peierls valleys, $b_{\mathrm{p}}$ the Burgers vector of partials, $\beta$ depends on the angle between $b_{\mathrm{p}}$ and the dislocation line, $d_{0}$ is the equilibrium distance of the two partials and $\gamma$, the stacking fault energy. Resulting values are listed in table IV, $\tau_{c}$ falls in the lower part of the « central » stress range.

Table IV. - Critical shearstress for the transition from correlated to uncorrelated kink pair formation on partial dislocations (after [40]). $\tau_{c}$ sensitively depends on $\gamma$ and the values listed here are somewhat higher than those of [40] due to the higher stacking fault energies determined recently and also to the use of averaged $\mu$ values (Voigt) instead of $\mu=\mathrm{C}_{44}$.

\begin{tabular}{|l|c|c|c|}
\hline Material & $\begin{array}{c}\gamma \\
\left(\mathrm{mJ} . \mathrm{m}^{-2}\right)\end{array}$ & $\begin{array}{c}\tau_{c} \text { screw } \\
(\mathrm{MPa})\end{array}$ & $\begin{array}{c}\tau_{c} 60^{\circ} \\
(\mathrm{MPa})\end{array}$ \\
\hline $\mathrm{Si}$ & $55-75$ & $9.8-18$ & $6.7-12.4$ \\
$\mathrm{Ge}$ & $71-85$ & $18.2-26$ & $12.7-18.2$ \\
$\mathrm{GaSb}$ & $46-60$ & $11-18.6$ & $7.1-12$ \\
$\mathrm{GaAs}$ & $42-60$ & $7.7-15.7$ & $5-10.1$ \\
$\mathrm{InSb}$ & $34-42$ & $7.7-11.8$ & $4.5-6.9$ \\
$\mathrm{GaP}$ & $37-45$ & $5.3-7.8$ & $3.5-5.15$ \\
$\mathrm{InAs}$ & $27-33$ & $4.4-6.6$ & $2.65-4$ \\
$\mathrm{InP}$ & $15-21$ & $1.3-2.5$ & $0.7-1.4$ \\
\hline
\end{tabular}

The theoretical analysis of dislocation motion taking the dissociation into account was done by Möller [40] in 1978, in the framework of models in fashion at that time, assuming a negligibly small $W_{\mathrm{M}}$ but with a temperature dependent concentration of localized obstacles controlling the average kink drift velocity. The results are complicated, with a lot of fitting parameters. The model successfully explains the marked increase of $Q$ at low stresses. It should be adapted to the present idea of a high secondary Peierls relief, which certainly screens out occasional weak obstacles.

According to this analysis, the high stress regime where dislocation motion is controlled by uncorrelated motion of partials appears to be simpler and would obey the equations given in \$ 3.1. However this high stress range was recently explored in great detail by Alexander et al. $[99,100]$ who showed that further complications arose. Two complementary approaches were used :

(i) velocities of the total dislocations were measured in very pure floating zone $\mathrm{Si}$;

(ii) from measurements of dissociation widths frozen-in under load, relative mobilities of the various partial dislocations were also obtained.

The results of Kiesielowski on total dislocation velocities are the following:

(i) the dependence of the velocity $v$ on the resolved shear stress $\tau$ can be described by the classical power law in the range $30 \mathrm{MPa} \leqslant \tau \leqslant$ $300 \mathrm{MPa}$ at $420^{\circ} \mathrm{C}$;

(ii) the parameters $v_{0}$ and $m$ depend, not only on the type of the dislocation, but also, for a given dislocation type, on the glide system to which the dislocation belongs $(1.2<m<2.2)$, which means that Schmid's law is not obeyed in Si i.e. the non shear component acts on the dislocation velocity.

(iii) An empirical expression is given for the stress exponent :

$$
m=m_{0}+m_{1}=-7.2(1-|\mathbf{b} \cdot \mathbf{c}|)^{2} F_{\mathrm{n}} / b \sigma+m_{1}
$$

where $m_{1}$ depends only on the dislocation type (character and order of partials), and $m_{0}$ is calculated from the angle between the Burgers vector $b$ and the stress axis c (unit vectors) and from the normal component $F_{\mathrm{n}}$ of the force acting on the dislocation, normalized by $b \sigma$ ( $\sigma$ : nominal applied stress).

On the other hand, Weiss reported that the dissociation widths of similar dislocations in different glide systems (in same $\tau, T$ conditions) could not be explained by mobility ratios which would depend only on the type and order of partial dislocations, first suggested by Wessel and Alexander [99]. In the steady state assumption, assuming a different lattice resistance for the two partials, the balance of forces acting on them yields for the dissociation width :

$$
d=d_{0} /\left[1-(f-\delta) \frac{\tau b}{2 \gamma}\right]
$$

where $d_{0}$ is the dissociation width at zero stress, $f=\left(F_{2}-F_{1}\right) /\left(F_{2}+F_{1}\right)$ and $\delta=R_{2}-R_{1} / R_{2}+R_{1}$, $F_{i}$ and $R_{i}$ being respectively the externally applied force and the total glide force (equal to the lattice resistance in the steady state) felt by the partial dislocation $i$. Heister of the same group assumed that the power law valid for the total dislocation was 
also obeyed by partial dislocations with the same $m$ exponent. He was then able to determine mobility ratios accounting for both the velocities measured by Kiesielowski and the dissociation widths observed by Weiss. The detailed analysis is given in [100]. The difficulty remains to give a physical meaning to $m$ and to justify the apparently inconsistent assumption that $m$ is the same for both partials, whereas a part of it depends on the dislocation character. Alexander argued that both partials could be concerned in the same way by the two effects that enter in $m:$ a reduction by the shear stress of the energy to be thermally activated and a possible modification of the prefactor (via detailed changes of the core structure).

Here again the rather large scatter in experimental data on dissociation widths must be recalled.

An exhaustive study of dissociation widths under stress has been conducted in Si by Demenet et al. $[101,102]$. All possible cases for the applied stress tensor on partial dislocations have been theoretically checked. Two kinds of situations were experimentally investigated: static and dynamic cases. In a static case shear stresses on partials are equal and opposite so that the dissociated configuration as a whole is at rest and the friction forces do not need be taken into account. Under these conditions one can distinguish two possibilities : one in which applied stresses are in the sense of a widening of the stacking fault ribbon and the reverse one.

imental results have been obtained since in the two situations, splitting widths are found to be larger than theoretically expected. In these two cases climb forces are quite large so that point defect interactions with dislocations could be responsible for such an effect.

Dynamical cases different from those investigated by the Alexander group have been studied, such as those where one of the two partials is submitted to a zero force, It seems that the whole set of results can only be explained if the climb force on each partial dislocation is taken into account not only through its magnitude (cf. Alexander group) but also through its direction.

5.2.3 Influence of the dislocation character. - According to results reported on above, it is clear that slight changes in $Q$ and $m$ for different dislocation characters should correspond to different velocities and to relative mobilities that vary as functions of $\tau$ and $T$. The differences between $v_{\mathrm{s}}$ and $v_{60}$ appear to be rather small in $\mathrm{Si}\left(0.5<v_{60} / v_{\mathrm{s}}<2\right.$ in the investigated range including high stresses) but can be larger in $\mathrm{Ge}\left(v_{\mathrm{s}} / v_{60} \cong 10\right.$ at $\tau=10 \mathrm{Mpa}, T \cong 370^{\circ} \mathrm{C}[78$, 79]). From the consideration of the core structure, it is expected that $30^{\circ}$ partials and $90^{\circ}$ partials behave differently. This should induce different behaviours for screws (made of two $30^{\circ}$ partials) and $60^{\circ} \mathrm{s}$ (made of one $30^{\circ}$ and one $90^{\circ}$ partial). According to Alexander and coworkers a given partial may behave differently depending on its position - leading or trailing - in the total dislocation. To sum up qualitatively Alexander group results, in $\mathrm{Si}$, the trailing $30^{\circ}$ partial in screws is less mobile than the leading one. In $60^{\circ}$ dislocations, the $90^{\circ}$ partial is more mobile than the $30^{\circ}$ partial whatever their leading or trailing respective character. The behaviour of $30^{\circ}$ partials in $60^{\circ}$ dislocations, leading or trailing, is the same as that of the leading one in screws.

Indeed $60^{\circ}$ dislocations of the $30^{\circ} / 90^{\circ}$ and $90^{\circ} / 30^{\circ}$ types have slightly but significantly different mobilities in $\mathrm{Si}$ and $\mathrm{Ge}$ even in the low stress range - not to speak about the further difficulties introduced by non shear stresses described in the previous section. In compounds, a further difference is expected from the $\alpha$ or $\beta$ character of partials (Fig. 5b). In these materials six types of dislocations should be considered. According to recent results in InP (S doped) these six dislocation types do have different velocities [88]. The influence of the $\alpha \beta$ or $\beta \alpha$ character was also observed to induce different abilities to cross-slip in GaAs by Sato et al. [52], and for screw dipoles to close up by Küsters et al. [51].

In III-V compounds however, the main result is the very large difference between the $60^{\circ} \alpha$ dislo-

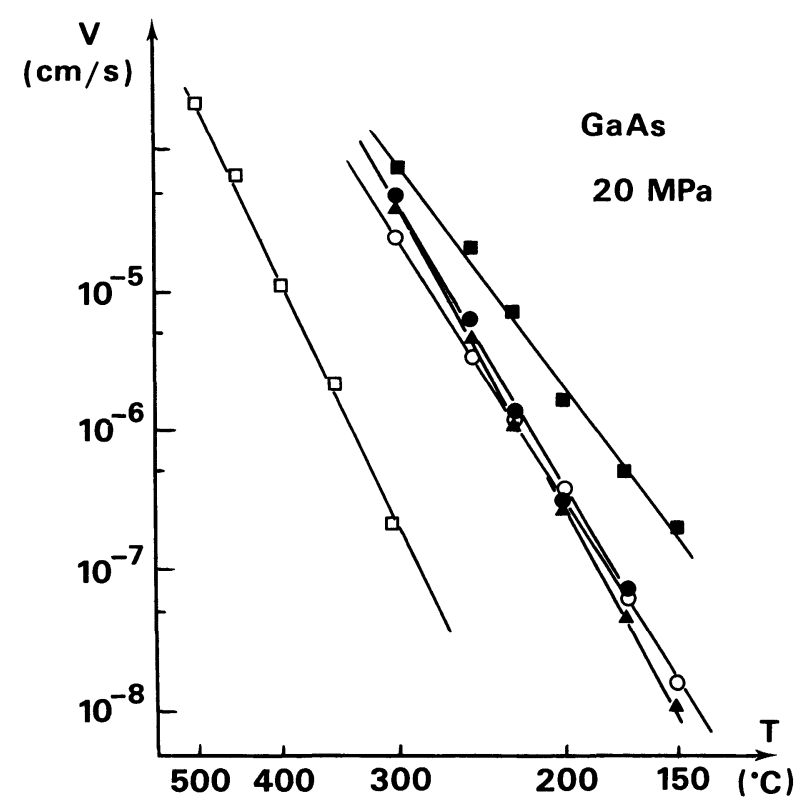

Fig. 14. - Velocities of $\alpha, \beta$ and screw dislocations in GaAs. Open symbols nominally undoped, n-type GaAs $\left(\sim 10^{17} \mathrm{~cm}^{-3}\right)$. Full symbols : $\mathrm{Zn}$ doped, p-type GaAs $\left(3 \times 10^{18} \mathrm{~cm}^{-3}\right)$ after [84].

$0,0: 60^{\circ} \alpha$ dislocations ;

$\square, \square: 60^{\circ} \beta$ dislocations ;

$\Delta$ : screw dislocations. 
cation and $60^{\circ} \beta$ or screw dislocations. In all known cases :

$$
v_{\alpha} \gg v_{\mathrm{s}}, v_{\beta}
$$

with a difference usually by more than one order of magnitude (Fig. 14). In most cases the sequence of partials is not given by authors but there are strong indications that the two types of $60^{\circ} \alpha$ dislocations are faster than the other four possible types [88].

5.3 THE DOPING EFFECT. - The very strong effect of electrically active impurities on dislocation mobili-
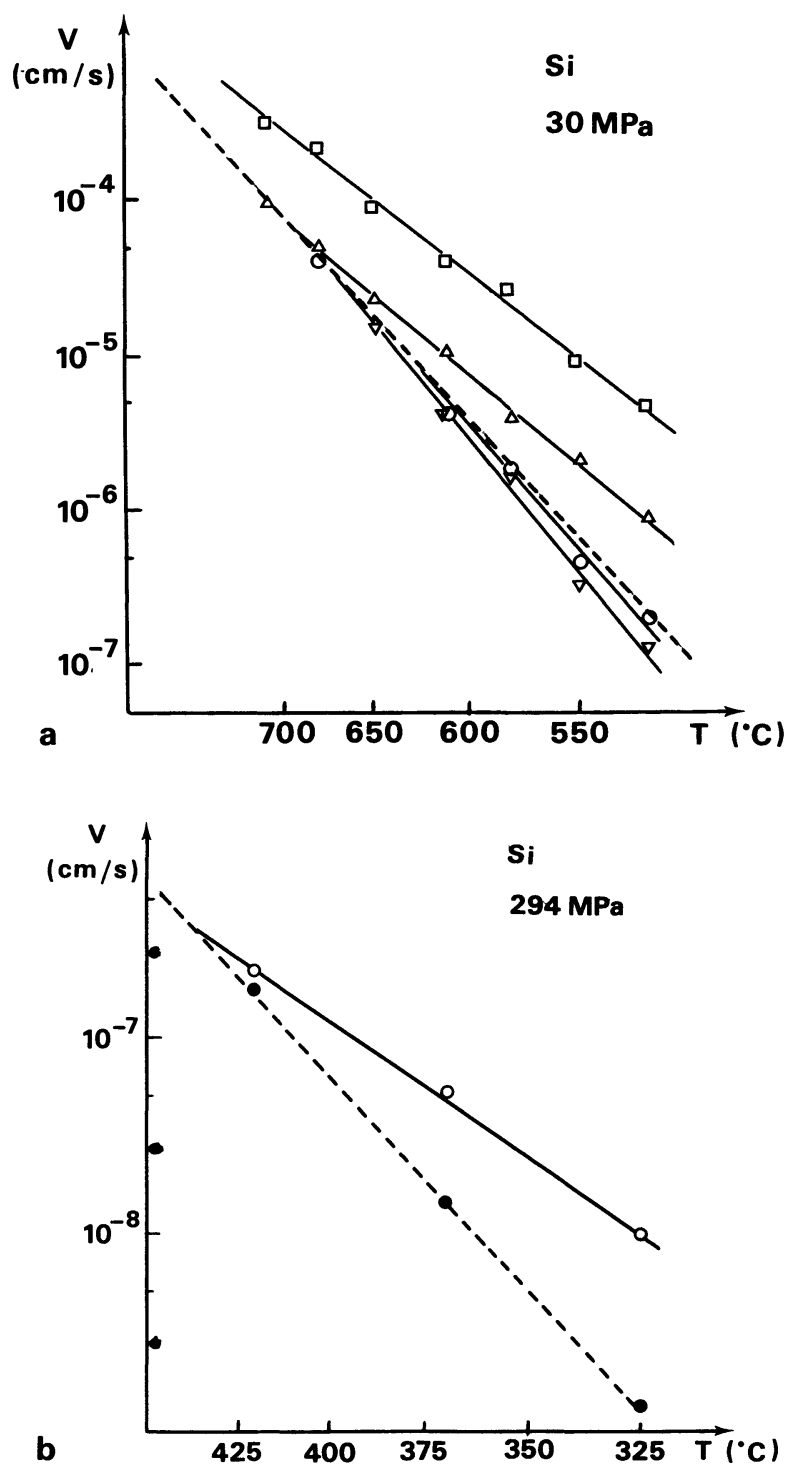

Fig. 15. - Change in dislocation velocities and apparent activation energies by doping, light illumination or electron irradiation. (a) Doping effect in $\mathrm{Si}$ after [74]. Open symbols : $60^{\circ}$ dislocations. Full symbols : screw dislocations. Dotted lines : intrinsic $\mathrm{Si}$

$\square, \square \mathrm{n}(\mathrm{P}) 1.4 \times 10^{19} \mathrm{~cm}^{-3}$

$\triangle, \Delta \mathrm{n}(\mathrm{P}) 1.2 \times 10^{18} \mathrm{~cm}^{-3}$

$\mathrm{O}, \bullet \mathrm{p}(\mathrm{B}) 2.7 \times 10^{17} \mathrm{~cm}^{-3}$

$\nabla, \nabla \mathrm{p}(\mathrm{B}) 6.8 \times 10^{17} \mathrm{~cm}^{-3}$ ty first observed by Patel and Chaudhuri [103] in Ge is now well established in most SC. The origin of this effect has been discussed in $\$ 4.2 .1$, let us recall here the main experimental features.

At temperatures where the number of extrinsic free carriers exceeds that of thermal carriers $\left(T \leq T_{\mathrm{d}}\right)$, the velocity of a dislocation can be either increased or decreased depending on the type of doping $-\mathrm{n}$ or $\mathrm{p}$-, the material under study and the doping concentration.

Formally, the dislocation velocity in doped materials obeys an Arrhenius law with different activation
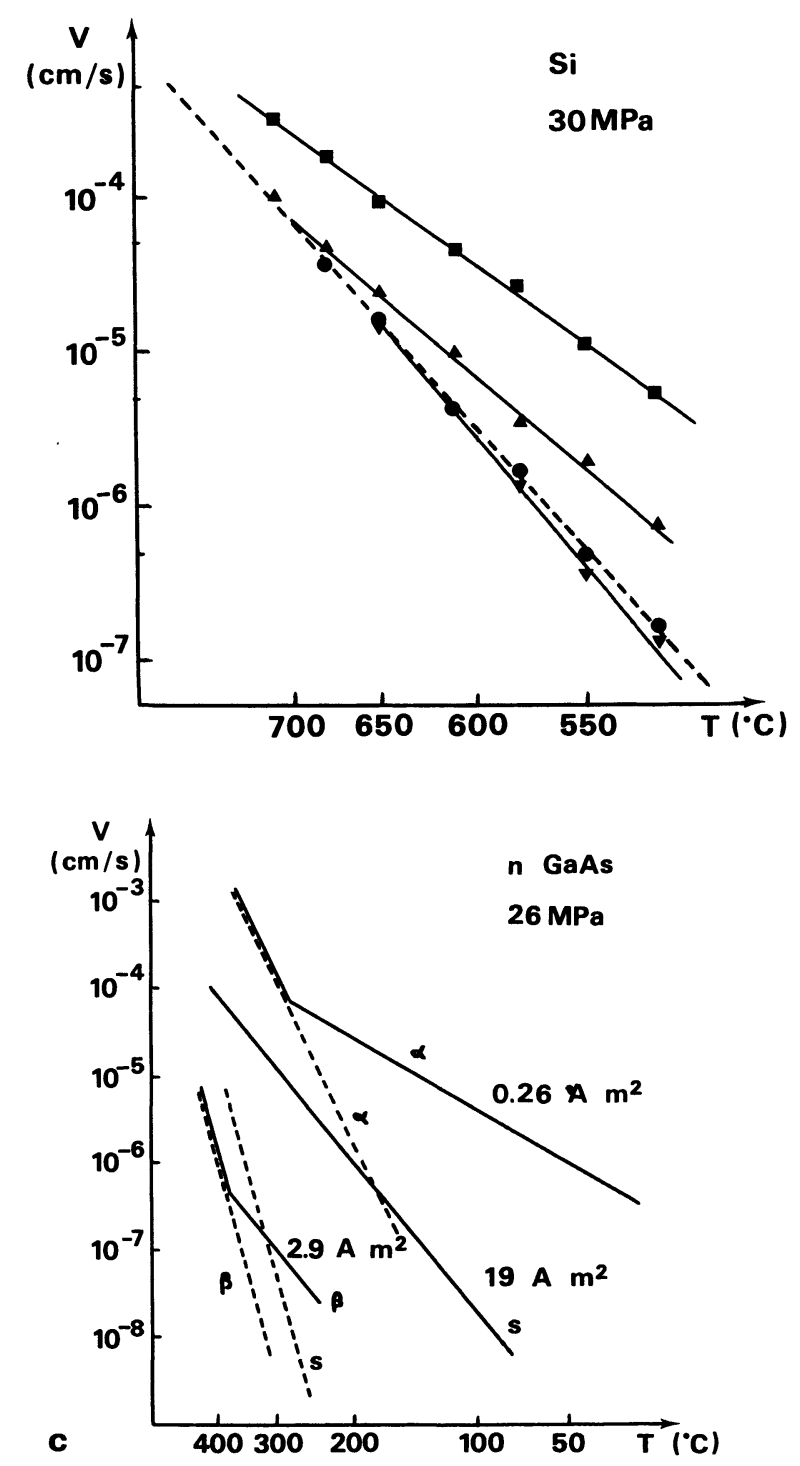

(b) Photoplastic effect in $\mathrm{Si}$ after [109]. $30^{\circ} / 90^{\circ}$ dislocations. Open symbols: velocities under illumination $\left(h \nu=1.17 \mathrm{eV}, P=1 \mathrm{~W} / \mathrm{cm}^{2}\right)$. Full symbols : velocities in the dark.

(c) Effect of irradiation by $30 \mathrm{keV}$ electrons in $\mathrm{n}-\mathrm{GaAs}$ $\left(1.4 \times 10^{18} \mathrm{~cm}^{-3}\right)$ after [110]. Full lines : velocities under irradiation (numerals are irradiation intensities). Dotted lines : velocities in the dark. 
energy and prefactor. An increase of $v$ is obtained by a decrease of $Q$, partially compensated by a decrease of the prefactor in order to make the velocities in doped and undoped materials coincident at $T \cong$ $T_{\mathrm{d}}$. A decrease of $v$ corresponds to higher $Q$ and prefactor (Fig. 15a). In the whole temperature range, experimental data can be described by the addition of two Arrhenius factors, each one becoming predominant in one of the two ranges separated by $T_{\mathrm{d}}$.

$$
v=v_{0}^{\mathrm{i}} \exp \left(-Q_{\mathrm{i}} / k T\right)+v_{0}^{\mathrm{d}} \exp \left(-Q_{\mathrm{d}} / k T\right)
$$

where the subscripts $i$ and $d$ are for intrinsic or doped respectively. As far as it has been studied, the stress dependence described by the exponent $m$ is not much affected by doping [74].

The doping effect does not depend on the nature of the doping atom but only on its donor or acceptor character. Same concentrations of $\mathrm{P}$ and $\mathrm{Sb}$ have the same effect in $\mathrm{Si}$ and the same holds true for $\mathrm{B}, \mathrm{Al}$ or $\mathrm{Ga}$ atoms. This statement is valid only when the doping effect is not obscured by «metallurgical » hardening effect (\$ 4.2.2). As far as dopants are considered, these effects can be neglected usually at concentrations lower than a few $10^{18}$ at. $\mathrm{cm}^{-3}$ since dopants are slow diffusors [104].

It may be important to notice that spurious effects may result from a change in the solubility of any neutral unwanted impurity by the type of doping. e same proce ure, one can observe accidental $\mathrm{Cu}$ contamination in p-type $\mathrm{Si}$ and not in n-type $\mathrm{Si}$, because $\mathrm{Cu}$ solubility is increased by $\mathrm{p}$-doping.

The doping effect is concentration dependent. Available results have been displayed in a schematic form in figure 16 for $\mathrm{Si}[74,75,105]$, Ge [103, 106], InSb [81, 106] and GaAs [84, 85, 107, 108]. These qualitative trends were established by several groups and can be taken for sure, although there are large discrepancies in the data collected in III-V compounds.

Striking is the fact that qualitatively the effect is varying from one material to the other. n-doping increases dislocation velocity in $\mathrm{Si}$ and $\mathrm{Ge}$ but rather decreases it in InSb and GaAs. p-doping markedly decreases the velocity of $60^{\circ}$ dislocations in Ge and has a very little effect on $60^{\circ}$ dislocations in Si. The doping effect may also be different for different types of dislocations. In Si the strong enhancement of mobility by n-doping is quite the same for screws and $60^{\circ}$. (It seems that only $60^{\circ}$ dislocations have been studied in Ge.) In CSC, on the other hand, $\beta$ dislocations seem to be much more sensitive to the doping than $\alpha$ dislocations. Especially, p-doping increases the velocity of $\boldsymbol{\beta}$ dislocations and leaves nearly unaffected that of $\alpha$ dislocations in GaAs. Thus, there is a range in p-type GaAs where the mobilities of these two dislocation types are nearly
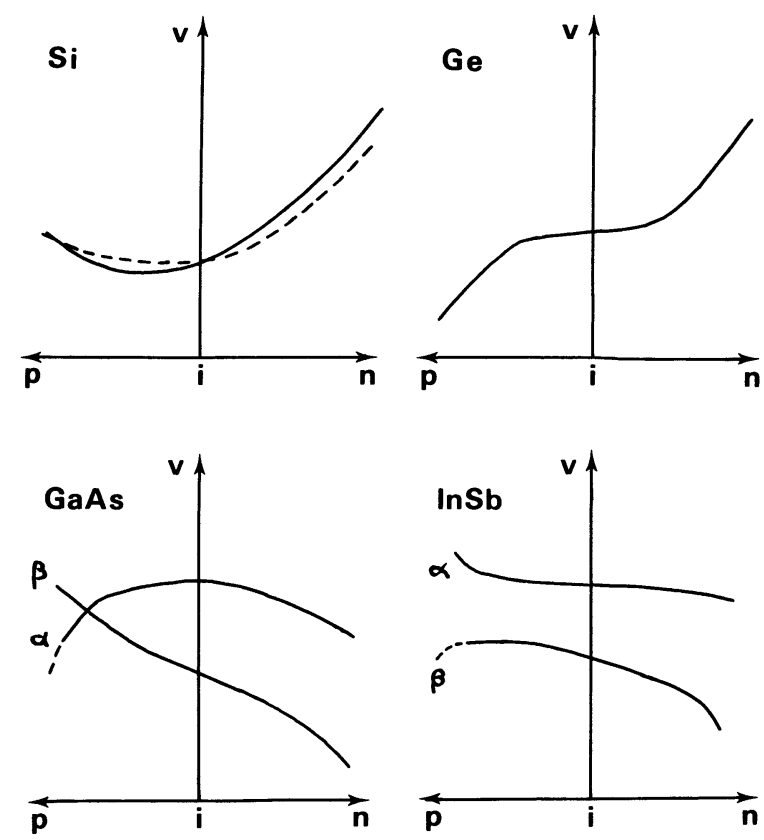

Fig. 16. - Dislocation velocities in semiconductors as a function of the doping concentration (schematic: the « intrinsic » range depends on temperature). After references given in the text.

equal, as in ESC. A similar situation was found in sulfur doped InP ( $\mathrm{n}$ type, $\sim 5 \times 10^{18}$ at $\mathrm{cm}^{-3}$ ) where $\alpha, \beta$ and screw mobilities are of the same order of

InP.)

A few remarks can be added to the theoretical discussion of $\S 4.2 .1$ :

(i) Basic mechanisms of the doping effect cannot be better established than those of dislocation glide in pure materials.

(ii) Whatever the mechanism involved, it must be recognized that in different undoped materials, dislocations are not necessarily in similar states of charge. This point would require a detailed investigation of their associated deep levels by electrical techniques, a picture which is far from being clear even for static dislocations in ESC ! Let us suggest that, if appropriate doping of each material could ensure similar charge for a given type of dislocation, correlations between the behaviours of different SC could become apparent, whereas they are hidden in undoped SC by different states of charge.

(iii) Clearly different from most impurity effects in metals, the doping effect could be thought to be rather similar to the interstitial softening observed in bcc metals. We feel however that origins are different. In SC, the mobility enhancement is due to a change in electrical properties, via a change of the Fermi level by shallow donors or acceptors. Contrarily to metals, the precise localization of the doping atoms with respect to the dislocation line is not important (cf. 4.2.2). 


\subsection{ENHANCEMENT OF DISLOCATION MOBILITY BY} OPTICAL EXCITATION. - The effect of illumination on dislocation velocities (photoplastic effect) has been demonstrated in Si by Küsters and Alexander [109] and the similar effect of electron irradiation in GaAs and InP by Maeda, Takeuchi and coworkers [110]. Very recently the photoplastic effect was reported by Takeuchi [111] in $\mathrm{Si}$, confirming Küsters results, but no effect has been found in $\mathrm{Ge}$ so far [111].

In $\mathrm{Si}$ a substantial enhancement of screw and $30^{\circ} / 90^{\circ}$ dislocations was observed at $T=437^{\circ} \mathrm{C}$, $\tau=300 \mathrm{MPa}$ under illumination by light with a photon energy just above the band gap. The velocity of $90^{\circ} / 30^{\circ}$ dislocations was not affected (Fig. 15b). The activation energy was reduced from the value observed in the dark by an amount of $0.68 \mathrm{eV}$ for $30^{\circ} / 90^{\circ}$ dislocations, $0.56 \mathrm{eV}$ for screws.

In GaAs (Si doped, $1.4 \times 10^{18} \mathrm{~cm}^{-3}$ ) an enhancement of all dislocation mobilities was observed under irradiation by $30 \mathrm{keV}$ electrons at $\tau=$ $26 \mathrm{MPa}$, at sufficiently low temperatures (Fig. 15c).

The reduction of activation energy under the electron beam is very large : about 0.7 to $0.9 \mathrm{eV}$ for $\alpha, \beta$ and screw dislocations in GaAs and for $\beta$ dislations in InP.

Formally the velocity under illumination can be described like the doping effect :

$$
v=v_{\mathrm{d}} \exp (-Q / k T)+\eta I \exp [-(Q-\Delta \varepsilon) / k T]
$$

where $Q$ is the activation energy in the dark and $\Delta \varepsilon$ the reduction of the activation energy under light which was observed not to depend on the beam intensity but which depends on the dislocation character. The prefactor of the second term varies linearly with the beam intensity, $I$. Because of this, the temperature at which the effect saturates for a given type of dislocation is shifted with the beam intensity.

The photoplastic effect was often claimed in the past, but the cause of the mobility enhancement was probably the heating by illumination. This trivial explanation can be ruled out now since it was checked very carefully by the authors mentioned above.

The photoplastic effect is presently ascribed to non-radiative electron-hole recombinations at dislocations, which are known to act as very active recombination centres at these temperatures. The vibrational energy released by the recombination can help either double kink nucleation or kink migration processes, provided associated energies are larger than the observed energy reduction. It is more likely that the recombination occurs at localized defects such as kinks, rather than on a defect-free dislocation line. This argument is in favour of an enhancement of the kink velocity, consistently with the present idea of a large $W_{\mathrm{M}}$.
5.5 EFFECT OF NON DOPING IMPURITIES ON DISLOCATION MOBILITY. - Direct evidence of impurity dislocation interaction in semiconductors was given thirty years ago by Dash who used copper decoration to image dislocations in $\mathrm{Si}$ slices [112]. Figure 17 shows an example of accidental decoration by metallic fast diffusors in $\mathrm{Si}$ [113].

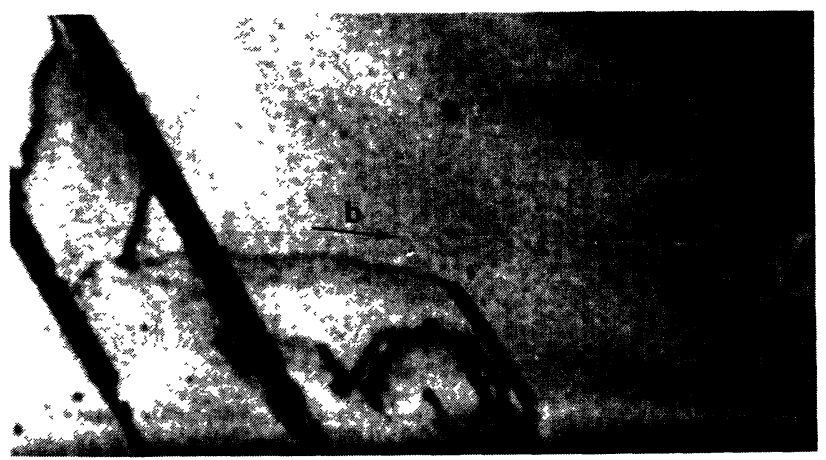

Fig. 17. - Dislocation loop decorated by metallic impurities in silicon. One $60^{\circ}$ segment only is heavily decorated. X-Ray topograph. Marker $200 \mu \mathrm{m}$ (A. George, unpublished).

Nowadays alloying with non doping impurities is a very important technique to improve the quality of semiconductor wafers which are submitted during device processing to a sequence of high temperature treatments with rapid heating and cooling cycles which generate thermal stresses.

It has long been recognized that Czochralski grown silicon (CZ-Si) has a better resistance to warpage, i.e. to dislocation slip caused by thermal stresses, than floating zone silicon (FZ-Si). The reason is the hardening effect of oxygen atoms that are present in appreciable concentrations $\left(\sim 3 \times 10^{17}\right.$ to $\sim 10^{18}$ at. $\left.\mathrm{cm}^{-3}\right)$ in the former material.

For the III-V compounds grown by the Czochralski pulling technique, alloying with an isovalent group III element is a now widely used technique to reduce the density of grown-in dislocations, which have a detrimental effect on device performances. In is added at "metallurgical " concentration $\sim 1 \%$ - to GaAs to obtain large diameter dislocation-free semi-insulating crystals [114]. Ga atoms have been used also to efficiently harden InP [115]. From the microscopic point of view, the relevant features of these hardening techniques must be revealed in the effect of impurities on dislocation mobility, which are described below. The influence of neutral impurities on the macroscopic mechanical behaviour will be treated in part II.

The influence of light element impurities, oxygen but also nitrogen and carbon, on dislocation mobility in $\mathrm{Si}$ was investigated in great detail by Sumino et 


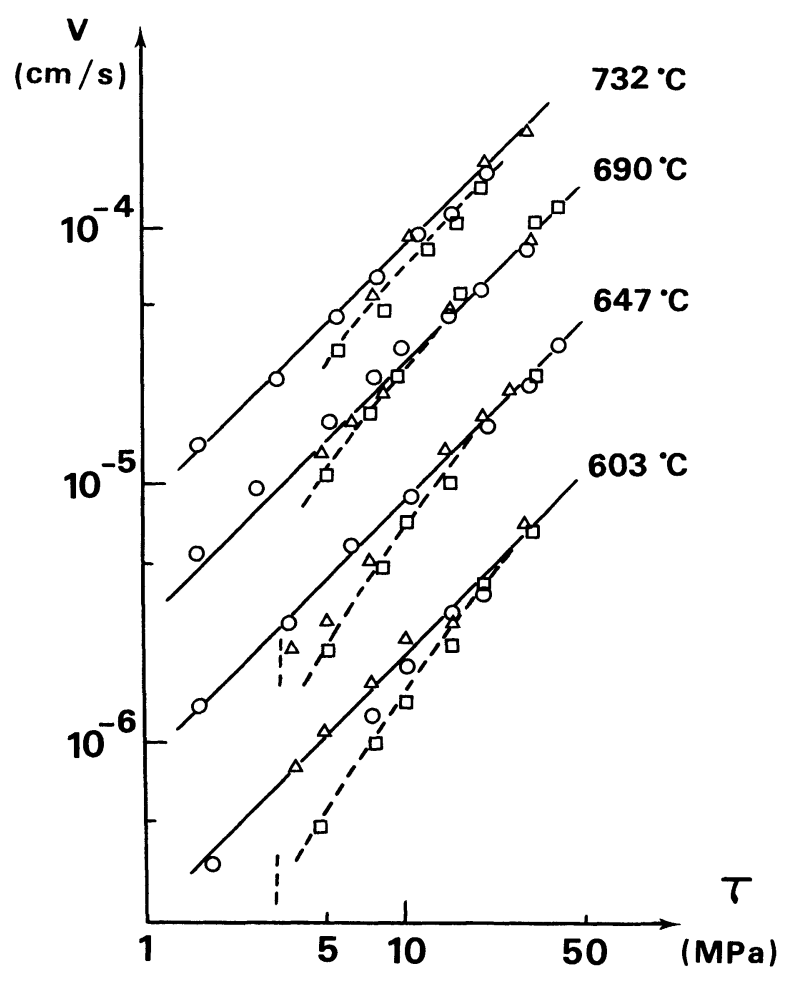

Fig. 18. - The effect of non electrically active impurities on $60^{\circ}$ dislocation velocity in silicon after [76]. Full lines : high purity FZ silicon (data points have been omitted for clarity).

O FZ Si containing $10^{17} \mathrm{C}$ atoms per $\mathrm{cm}^{3}$.

$\triangle$ FZ Si containing $5.4 \times 10^{15} \mathrm{~N}$ atoms per $\mathrm{cm}^{3}$.

$\square \mathrm{CZ} \mathrm{Si}$ containin 7.4

al. [80, 116-118]. Their results for $60^{\circ}$ dislocations are presented in figure 18 . Dislocation velocities in very pure FZ Si can be described by the usual power law stress dependence down to stresses $\tau \sim 2 \mathrm{MPa}$. In crystals containing oxygen, dislocations move at velocities which are ermo those in the high purity crystal in the high stress range. Below a given stress value a departure from the power law dependence is observed with lower average mobilities. If the stress is further decreased dislocations cease to move. The deviation of the velocity from that in high purity crystals and the critical stress for immobilization both increase when the oxygen concentration increases. The critical stress for immobilization is $\sim 3 \mathrm{MPa}$ at $[0]=15$ at . ppm and $\sim 7 \mathrm{MPa}$ at [0] $=$ 18 at. ppm. In FZ Si containing a rather low concentration of $\mathrm{N}$ atoms, $\sim 0.1$ at. ppm, dislocations become immobile at $\tau \leqslant 4 \mathrm{MPa}$ and move with the same mobility as in pure Si at $\tau>4 \mathrm{MPa}$. Nitrogen atoms have a stronger pinning effect than oxygen atoms. $\mathrm{C}$ atoms at a concentration of 2 at.ppm have no effect on dislocation mobility. Similar results hold for screw dislocations.

$\mathrm{X}$-ray topography revealed that the shape of moving dislocations is perturbed from the regular (semi-) hexagon with $\langle 110\rangle$ straight segments in the intermediate stress range when the velocity deviates from that in pure Si. Then moving dislocations become irregular i.e. the velocity is no more constant along the dislocation line. These perturbations however are essentially reversible and loops catch up their regular shape when submitted to high stress again. On the other hand, once dislocations have become immobile in the impure crystals, the stress needed to put them in motion - starting stress increases with the period and the temperature where dislocations have been kept at rest, depending on the impurity atom and the concentration involved. See [116] for a quantitative study of the starting (or locking) stress as a function of ageing and impurity concentrations. Sumino states that the starting stress is determined by the number of impurity atoms that have been gathered on a unit length of dislocation for a given initial concentration. It is suggested that dislocations interact with clusters of oxygen atoms rather than with individual atoms.

Yonenaga, Sumino and Yamada [119] very recently measured the mobilities of $\alpha, \beta$ and screw dislocations in Indium alloyed $\mathrm{GaAs}$ and compare them to those in Si doped and undoped GaAs. They observed that at $\tau=20 \mathrm{MPa}, \mathrm{T} \sim 450^{\circ} \mathrm{C} \mathrm{Si}$ doping is much more efficient at reducing the velocities of $\alpha$ and $\beta$ dislocations than In addition. However the two effects are qualitatively different. Si has a doping effect : the velocity is decreased by about one a concentration of $7 \times$ $10^{-3} \mathrm{ppm}$ but dislocations are still mobile at very low stresses ( $\cong 2 \mathrm{MPa})$. In at much higher concentration has a small effect on dislocations below $\sim 20 \mathrm{Mpa}$ and immobilizes them at $\tau \leqslant 10 \mathrm{MPa}$. The behaviour is more similar to that of oxygen in Si. The locking stress is also observed to increase with increasing temperature and period of ageing. It may be remarked that if screw dislocations behave like $\alpha, \beta$ dislocations seem to be unaffected by In, which suggests that In mainly interacts with partial dislocations of $\alpha$ character.

A strong locking effect was also observed for $\mathrm{Ga}$ in InP [120].

5.6 ASYMMETRY OF DISLOCATION VELOCITY. - To stress the point that many aspects of dislocation mobility are still far from being well understood, let us mention to conclude this section the asymmetry reported by Nikitenko and coworkers [121-122] for the velocity of dislocations in $\mathrm{Si}$ and $\mathrm{Ge}$ when the applied stress was reversed. As a result, reversal of the stress increases the dislocation velocity substantially, by up to two orders of magnitude. In these experiments the stress was applied by four point bending and could be reversed without intermediate cooling using a six support bending jig to transfer the loading from the inner supports to the extreme ones.

The difference between dislocation velocities in 
opposite glide directions was found to be very sensitive to the temperature, stress and heat treatment of the crystal prior to reversal of the stress sign. At low temperature $\left(T \leqslant 700^{\circ} \mathrm{C}\right.$ in $\left.\mathrm{Si}\right)$ the increase of $v$ can be described as the result of a decrease of $Q$ (from $2.3 \mathrm{eV}$ to $\sim 1.5 \mathrm{eV}$ in extreme cases). The asymmetry is stronger without intermediate cooling and vanishes after a sufficient annealing time. At higher temperature the difference in forward and backward velocities decreased more rapidly than predicted by the Arrhenius law with the modified $Q$, especially after some intermediate cooling or annealing. The asymmetry magnitude is reported to decrease as the stress increases and examples shown are for small stress values $(\tau \sim 5 \mathrm{MPa}$ in $\mathrm{Si})$, very close to the critical stress for correlated double kink formation on partials.

Those authors found no difference in the dissociation width or character depending on the sense of motion. The asymmetry was observed in doped crystals as well. When the cycle is repeated, the forward motion of dislocation is characterized by the same velocity and activation energy as in the case of the first half-loop extension.

Such an effect recalls the long observed very fast shrinkage of small dislocation loops, in the absence of applied stress but under the action of the line tension $[74,79]$. However the effect of line tension i.e. the large back stress at dislocation bends which should promote kink formation at these points, in the backward motion, is ruled out by Nikitenko and coworkers who used loops of large diameter. It is indeed difficult to accept that the overall dislocation velocity can be controlled by easy kink formation at four special points (inner bends and surface points) of a dislocation line with the experimental evidence of a high energy for kink migration. The argument however should be quantitatively checked. On the other hand, some of the observed features - such as the suppression of the asymmetry by annealing can be explained by impurity segregation to the dislocation. It is indeed conceivable that impurities form an atmosphere of defects that would be dense enough to impede long range motion of kinks formed at the weak dislocation points, but sufficiently dilute not to affect the forward velocity. The latter condition requires that the average spacing of impurity atoms along the dislocation line is larger than the kink mean free path in the conditions of forward motion.

The asymmetry was ascribed by Nikitenko and coworkers to interaction with point defects left in the wake of moving dislocations, in agreement with investigations of deformed crystals by EPR or DLTS techniques ( $\$ 4)$ and the observation by the Russian group of etch traces along the swept dislocation slip planes. However, it is difficult to understand how, in its back motion, a dislocation could absorb all defects previously emitted without creating any new ones, which assumption seems to be necessary to account for the fact that the velocity is not increased at all during a second movement in forward direction.

The asymmetry of dislocation velocity under stress reversal could also be of a similar nature as an other observation reported long ago [90] but not yet explained and which could be called « glide instabilities » : in these experiments, some parts of the dislocation loops were observed to move with a higher velocity than other segments of the same. crystallographic character which were moving simultaneously in the same sample under a constant applied stress. Such accelerating effects are not likely to be due to impurities, to which any slow down can always be ascribed, but raise the speculation about the possible co-existence of different core structures (glide $\rightleftarrows$ shuffle transformation). This question has been recently reinvestigated in detail by Louchet and Thibault-Desseaux [12] to whom the reader is referred.

\section{Conclusions.}

Dislocations in ESC and CSC with zinc blende structure show analogy with fcc metals as far as burgers vectors, slip geometry and dissociation are concerned. Specific features of dislocations in SC appear at temperature lower than $0.6 T_{\mathrm{m}}$. Dislocation movement is controlled by a Peierls mechanism reflecting the strong covalent bonding of the atoms. Deep electronic levels are associated with dislocations in the band gap and the glide behaviour can be changed with the electronic state of the crystal : doping element, carrier injection, illumination...

Although dislocations in ESC have been extensively studied (mainly in $\mathrm{Si}$ ) controversial points still remain in the explanation of specific features of dislocation glide such as : point defect interaction with dislocations during glide, influence of the nonshear component of the stress tensor on dislocation mobilities... The fact that such problems are not raised till now in CSC reflects quite obviously the fact that less sophisticated studies have been conducted on dislocations in III-V compounds, due certainly to the greater difficulty to get and handle samples of a suitable quality. There is a need to characterize more precisely dislocations in CSC at a microscopic scale.

Among principal trends the following points could be stressed :

Dislocations in ESC and CSC are dissociated when moving. This does not preclude that dislocations can actually be moving in the shuffle set. 
This possible change in the core structure via a glideshuffle transition has to be thought together with the occurrence of point defects left behind by dislocations by a mechanism which still needs to be elucidated. TEM studies of dislocation splitting in $\mathrm{Si}$ and $\mathrm{Ge}$ have shown a particular effect of thermal treatments on the splitting of screw dislocations. Populations of point defects are expected to be modified by those treatments, which suggests a modification of dislocation cores through their interaction with point defects. However this can be attributed to intrinsic point defects as well as extrinsic ones. The same comment could be made about the asymmetry of dislocation velocities when the stress is reversed.

Dislocation glide studies in ESC have high-lighted a strong secondary Peierls potential which can be explained by reconstruction of dangling bonds occuring in both partial dislocations and kinks. Breaking these bonds to move the dislocation is difficult, but the creation and the migration of such kinks can be explained through the existence of reconstruction defects, called antiphase defects or solitons. In this scheme the electronic doping effect is attributed to changes in the Fermi level causing changes in the concentrations and migration energies of charged elementary defects on dislocation lines. These defects can be kinks, solitons, kink-soliton complexes, following the various theories. Such theories could $s$ an in $s$ were reconstructed also in compounds. Such reconstruction can be more difficult as the ionic character of the bond increases. To confirm such hypothesis in CSC, determinations of the secondary Peierls potential are needed, for example by the determination of the critical length above which the velocity of a dislocation segment is not length dependent, which could be obtained by in situ TEM experiments.

Dislocation velocities have been determined rather accurately by several groups, especially in ESC, in a rather narrow range of temperature and stress-typically $0.45 T_{\mathrm{m}}-0.65 T_{\mathrm{m}} ; 5 \times 10^{-5} \mu-10^{-3} \mu$. Extensions of the investigated range yielded new and sometimes surprising effects such as the change in the apparent activation energy at very high temperatures or Schmid's law failure at large stress and the asymmetry after stress reversal. Several of these effects are not second order, but very large ones. It is desirable that they are confirmed by other groups and the improvement of experimental facilities should make experiments easier. For example, the dream of an ideal in situ - real time - observation of the response of dislocations in the bulk to stresses applied in tension or compression with the possibility of rapid stress-reversal, with the further possibility to modulate the stress application through pulse trains of variable durations, could become real soon thanks to the new dedicated synchrotron radiation facilities. It requires however a major effort to improve existing high temperature deformation stages and the collaboration of several $o$ care y check the impurity content (electrically active or not) of samples prior to, as well as after, observations.

\section{References}

[1] Alexander, H. and HaAsen, P., Solid State Physics, Seitz, F. et al. Edts, 22 (1968) 27

[2] Phillips, J. C., Bonds and Bands in Semiconductors (Academic Press, N. Y.) 1973.

[3] Chalmers, B. and Martius, U. M., Proc. R. Soc. A 213 (1952) 175.

[4] Ray, I. L. F. and Cockayne, D. J. H., Proc. $R$. Soc. A 325 (1971) 513.

[5] Cockayne, D. J. H. and Hons, A., J. Physique Colloq. 40 (1979) C6-11.

[6] Gottschalk, H., Patzer, G. and Alexander, H., Phys. Status Solidi A 45 (1978) 207.

[7] Meingast, R. and Alexander, H., Phys. Status Solidi (a) 17 (1973) 229.

[8] Gomez, A., Hirsch, P. B., Philos. Mag. 36 (1977) 266.

[9] Weber, E. and Alexander, H., Inst. Phys. Conf. Ser. 31 (1977) 266.

[10] Kimerling, L. C. and Patel, J. R., Appl. Phys. Lett. 34 (1979) 73.

[11] KisielowsKi-Kemmerich, C. and AleXander, H., in V Int. Symp. « Structure and Properties of dislocations in semiconductors », Moscou USSR, 1986.

[12] Louchet, F. and Thibault-Desseaux, J., Revue Phys. Appl. 22 (1987) 807.

[13] Tafto, J. and Spence, J. C. H., J. Appl. Cryst. 15 (1982) 60.

[14] Hünfeld Conference, J. Physique Colloq. 40 (1979) C6.

[15] Marklund, S., Phys. Status Solidi b, 92 (1979) 83

[16] Marklund, S., Phys. Status Solidi b, 100 (1980) 77.

[17] Jones, R., J. Physique Colloq. 40 (1979) C6-33.

[18] Jones, R., Oberg, S. and Marklund, S., Philos. Mag. B 43 (1981) 839.

[19] Cullis, A. G., J. Microsc. 98 (1973) 191.

[20] Gomez, A., Cockayne, D. J. H., Hirsch, P. B. and VITEK, V., Philos. Mag. 31 (1975) 105.

[21] Spence, J. C. H. and Kolar, H., Philos. Mag. A 39 (1979) 59.

[22] Foll, H. and Carter, C. B., Philos. Mag. A 40 (1979) 497.

[23] Alexander, H., J. Physique Colloq. 40 (1979) C61. 
[24] Chiang, S. W., Carter, C. B. and Kohlstedt, D. L., Philos. Mag. A 42 (1980) 745.

[25] Haussermann, F. and Schaumburg, H., Philos. Mag. 27 (1973) 745.

[26] Parckeiser, G. and HaAssen, P., Philos. Mag. 35 (1977) 821.

[27] Bourret, A. and Desseaux, J., Philos. Mag. A 39 (1979) 405.

[28] Pirouz, P., Cockayne, D. J. H., Sumida, N., Hirsch, P. B. and LANG, A. R., Proc. R. Soc. A 386 (1983) 241.

[29] Gomez, A. and Hirsch, P. B., Philos. Mag. A 38 (1978) 733.

[30] Feuillet, G., M. Sc. Thesis (1982) Oxford.

[31] Ourmazd, A., Cherns, D. and Hirsch, P. B., Inst. Phys. Conf. Ser. 60 (1981) 39.

[32] Cherns, D., Feuillet, G., Philos. Mag.

[33] Escaig, B., in Dislocation Dynamics, AR Rosenfield et al. Eds (Mc Graw Hill, London) 1968, p. 655.

[34] Siethoff, H. and Schroter, W., Zeit. Metallk. 75 (1984) 475.

[35] HiRTh, J. P. and Lothe, J., Theory of Dislocations (John Wiley \& Sons, New York) 1982.

[36] Marklund, S., Solid State Commun. 54 (1985) 835.

[37] Louchet, F., Inst. Phys. Conf. Ser. 60 (1981) 35.

[38] Hirsch, P. B., Ourmazd, A., Pirouz, P., Inst. Phys. Conf. Ser. 60 (1981) 29.

[39] Rybin, V. V. and Orlov, A. N., Sov. Phys. Solid State 11 (1970) 2635.

[40] Möller, H. J., Acta Metall. 26 (1978) 963.

[41] Jones, R., J. Physique Colloq. 44 (1983) C4-61.

[42] Fnaiech, M., Reynaud, F., Couret, A. and Caillard, D., Philos. Mag. A 55 (1987) 405.

[43] Esnouf, C., in Dislocations 84, Veyssière P., Kubin L. and Castaing J. Eds (CNRS Paris) 1984, p. 179.

[44] Hirsch, P. B., J. Physique Colloq. 40 (1979) C6117.

[45] HiRsCh, P. B., in Defects in semiconductors, Narayan and Tan Eds (North Holland, New York) 2 (1981) 257.

[46] Jones, R., Philos. Mag. B 42 (1980) 213.

[47] Heggie, M. and Jones, R., Philos. Mag. B 48 (1983) 365

[48] Heggie, M., in Dislocations 84, P. Veyssière et al. Eds (ed. CNRS Paris) 1984, p. 305.

[49] Kesteloot, R., Thèse $3^{\text {e }}$ cycle Lille (1981).

[50] Di Persio, J. and Kesteloot, R., J. Physique Colloq. 44 (1983) C4, 469.

[51] Kuesters, K. H., De Cooman, B. C. and Carter, C. B., Philos. Mag. A 53 (1986) 141.

[52] Sato, M., TAKebe, M., Sumino, K., in Dislocations in Solids, Yamada conference IX, H. Suzuki, T. Ninomiya, K. Sumino and S. Takeuchi Eds. (University of Tokyo Press) 1985, p. 429.

[53] Schröter, W., Scheibe, E. and Schoen, H., J. Micros. 118 (1980) 23.

[54] Kveder, V. V., Osipyan, Yu., A., Schroter, W. and Zoth, G., Phys. Status Solidi A 72 (1982) 701.

[55] Bauman, F. H. and Schroter, W., Philos. Mag. B 48 (1983) 55.
[56] Patel, J. R. and Kimerling, L. C., J. Physique Colloq. 40 (1979) C6-67.

[57] ARISTOV, V. V., ZOLOTUKHIN, M. N., KVEdeR, V. V., OsIPYAN, Yu. A., SNIGHIREVA, I. I. and Khodos, I. I., Phys. Status Solidi A 76 (1983) 485.

[58] Hirsch, P. B., J. Physique Colloq. 40 (1979) C6-87.

[59] Bretheau, T., Castaing, J., Rabier, J. and Veyssiere, P., Adv. Phys. 28 (1979) 829.

[60] Hutchison, P. W. and Dobson, P. S., Philos. Mag. A 41 (1980) 60.

[61] Petroff, P. M., J. Physique Colloq. 40 (1979) C6201.

[62] Weber, E. R., EnNen, H., Kaufmann, U., WINDS-CHEIF, J., SCHNEIDER, J. and WOSINSKI, J., J. Appl. Phys. 53 (1982) 6140.

[63] Martin, G. M. and MaKram-Ebeids, Physica 1163 (1983) 371.

[64] Figielski, T., Wosinski, T. and Morawski, A., J. Colloq. Physique 44 (1983) C4-353.

[65] Gruson, B. and FarvacQue, J. L. in V Int. Symp. «Structure and properties of dislocations in semiconductors » Moscou USSR (1986).

[66] Haasen, P., Phys. Status Solidi a 28 (1975) 145.

[67] HaAsen, P., J. Physique Colloq. 40 (1979) C6-111.

[68] Hirsch, P. B., J. Physique Colloq. 44 (1981) C3149.

[69] Puls, M. P., Philos. Mag. A 51 (1985) 893.

[70] Fleischer, R. C., Acta metall. 10 (1962) 3504.

[71] Skrotski, W. and HaAsen, P., J. Physique Colloq. 44 (1981) C3-119.

[72] Kubin, L. P., Rev. Def. Behav. Materials, P. Feltham ed. 4 (1982) 181.

[73] Brion, H. G., HaAsen, P. and Siethoff, H., Acta Metall. 19 (1971) 283.

[74] George, A., Champier, G., Phys. Status Solidi (a) 53 (1979) 529.

[75] Erofeev, V. N., Nikitenko, V. I., Sov. Phys. Solid State 13 (1971) 116.

[76] Imai, M., Sumino, K., Philos. Mag. A 47 (1983) 599.

[77] Kabler, M. N., Phys. Rev. 131 (1963) 54.

[78] Patel, J. R., Freeland, P. E., J. Appl. Phys. 42 (1971) 3298.

[79] Schaumburg, H., Philos. Mag. 25 (1972) 1429.

[80] Steinhardt, H., SChäFer, S., Acta Metall. 19 (1971) 65.

[81] Erofeeva, S. A., Osipyan, Y. A., Sov. Phys. Solid State 15 (1973) 538.

[82] Mihara, M., Ninomiya, T., Phys. Status Solidi (a) 32 (1975) 43.

[83] OsvenskiI, V. B., Kholodnyi, L. P., Sov. Phys. Solid State 14 (1973) 2822.

[84] Choi, S. K., Mihara, H., NinomiYA, T., Jpn. J. Appl. Phys. 16 (1977) 73.

[85] Steinhardt, H., HaAsen, P., Phys. Status Solidi (a) 49 (1978) 93.

[86] Choi, S. K., Mihara, M., Ninomiya, T., Jpn. J. Appl. Phys. 17 (1978) 32.

[87] Maeda, K., Takeuchi, S., Appl. Phys. Lett. 42 (1983).

[88] George, A., Jacques, A., Coquillé, R., Inst. Phys. Conf. Ser. 76 (1985) 439. 
[89] Chaudhuri, A. R., Patel, J. R., Rubin, L. G., J. Appl. Phys. 33 (1962) 2736.

[90] George, A., J. Physique colloq. 40 (1979) C6-133.

[91] Sumino, K., Harada, H., Philos. Mag. A 44 (1981) 1319.

[92] Di PERSIO, J., unpublished results in InSb.

[93] Louchet, F., Philos. Mag. A 43 (1981) 1289.

[94] Sato, M., Sumino, K., Proc. 5th Int. Conf. on HVEM (Kyoto, Imamura, T. and Hashimoto, H. Eds) 1977, p. 459.

[95] Farber, B. Ya., Nikitenko, V. I., Phys. Status Solidi (a) 73 (1982) K 14.

[96] FARber, B. Ya., Bondarenko, I. E., Nikitenko, V. I., Sov. Phys. Solid State 23 (1981) 1285.

[97] Gilman, J. J., J. Appl. Phys. 46 (1975) 5110.

[98] FARber, B. Ya., IUnin, Yu. L., Nikitenko, V. I., Phys. Status. Solidi (a) 97 (1986) 469.

[99] Alexander, H., in Dislocations 1984, Veyssière P., Kubin L., Castaing J. Eds (Editions du CNRS Paris 1984, p. 283.

[100] Wessel, K., Alexander, H., Philos. Mag. 35 (1977) 1523.

[101] Grosbras, P., Demenet, J. L., Garem, H., Desoyer, J. C., Phys. Status Solidi (a) 84 (1984) 481.

[102] DEMENET, J. L., unpublished results.

[103] Patel, J. R., Chaudhuri, A. R., Phys. Rev. 143 (1966) 601.

[104] Shaw, D., Phys. Status Solidi (b) 72 (1975) 11.

[105] Patel, J. R., Testardi, L. R., Freeland, P. E., Phys. Rev. B 13 (1976) 3548.

[106] Pirouz, P., Freeland, P. E., cited b ., ater. Sci. Res. 18 (1985) 1.
[107] NinOmyia, T., J. Physique colloq., 40 (1979) C6143.

[108] WARREN, P. D., private communication.

[109] Küsters, K. H., Alexander, H., Physica 116B (1983) 594.

[110] Maeda, K., Takeuchi, S., J. Physique Colloq. 44 (1983) C4-375.

[111] TAKEUCHI, S., Proc. 5th Int. Symp. « Structure and Properties of Dislocations in semiconductors » Moscou (1986) to be published.

[112] Dash, W. C., J. Appl. Phys. 27 (1956) 1193.

[113] GEORGE, A., unpublished results.

[114] JaCOB, G., DuseauX, M. Farges, J. P., VAN DEN Boom, M. M. B., RoksNoer, P. J., J. Cryst. Growth 70 (1984) 169.

[115] Peyrade, J. P., Coquillé, R., private communication.

[116] Sumino, K., Imai, M., Philos. Mag. A, 47 (1983) 753.

[117] Sumino, K., Yonenaga, I., Imai, M., Abe, T., J. Appl. Phys. 54 (1983) 5016.

[118] Sumino, K., Mater. Res. Soc. Symp. Proc. 14 (1983) 307.

[119] Yonenaga, I., Sumino, K., Yamada, K., Appl. Phys. Lett. 48 (1986) 326.

[120] George, A., Jacoues, A., Proc. 5th Int. Symp. «Structure and Properties of dislocations in semiconductors » Moscou (1986) to be published.

[121] Nikitenko, V. I., FARber, B. Ya., YAKIMOV, E. B., JETP Lett. 34 (1981) 232.

B., J. Physique colloq. ' 44 (1983) C4-85. 